\title{
Second-Order Sliding Mode Disturbance Observer-Based Adaptive Fuzzy Tracking Control for Near-Space Vehicles with Prescribed Tracking Performance
}

\author{
Bin Fu $(\mathbb{D}$, Kang Chen $(\mathbb{D}$, and Hang Guo \\ School of Astronautics, Northwestern Polytechnical University, Xi'an, China \\ Correspondence should be addressed to Kang Chen; mars_legend@163.com
}

Received 8 April 2018; Accepted 29 May 2018; Published 10 July 2018

Academic Editor: Luis J. Yebra

Copyright (C) 2018 Bin Fu et al. This is an open access article distributed under the Creative Commons Attribution License, which permits unrestricted use, distribution, and reproduction in any medium, provided the original work is properly cited.

\begin{abstract}
An adaptive fuzzy fault-tolerant tracking controller is developed for Near-Space Vehicles (NSVs) suffering from quickly varying uncertainties and actuator faults. For the purpose of estimating and compensating the mismatched external disturbances and modeling errors, a second-order sliding mode disturbance observer (SOSMDO) is constructed. By introducing the norm estimation approach, the negative effects of the quickly varying multiple matched disturbances can be handled. Meanwhile, a hierarchical fuzzy system (HFS) is employed to approximate and compensate the unknown nonlinearities. Several performance functions are introduced and the original system is transformed into one incorporating the desired performance criteria. Then, an adaptive fuzzy tracking control structure is established for the transformed system, and the predefined transient tracking performance can be guaranteed. The rigorous stability of the closed-loop system is proved by using the Lyapunov method. Finally, simulation results are presented to illustrate the effectiveness of the proposed control scheme.
\end{abstract}

\section{Introduction}

As is well known, disturbances and uncertainties widely exist in industrial systems and may induce negative effects on control performance or even stability of practical control systems [1]. Therefore, a wealth of disturbance and uncertainty estimation and rejection methods, such as unknown input observer (UIO) [2,3], perturbation observer [4], uncertainty and disturbance estimator [5], and disturbance observer (DO) [6], have been proposed. Meanwhile, a disturbance observer in frequency domain was developed in [7]. For the purpose of suppressing the periodic-disturbances, an adaptive periodic-disturbance observer is designed in [8]. In [9], a nonlinear disturbance observer is utilized in the robust control for spacecraft formation flying. In [10], a disturbance observer-based controller is developed for a magnetically suspended wheel with synchronous noise. In [11], a disturbance observer-based robust approaching control law is proposed for a tethered space robot. In [12, 13], several adaptive composite antidisturbance control methods have been investigated. In [14], a practical control method which can estimate the lumped disturbances consisting of both unknown uncertainties and external disturbances, called active disturbance rejection control (ADRC), was developed by Han and his collogues. Moreover, by using the equivalentcontrol-principle of the sliding mode control technique to estimate the lumped disturbances, sliding mode disturbance observer (SMDO) has been constructed [15]. In [16], to address the problem of disturbance rejection control for Markovian jump linear systems with matched and mismatched disturbances, an extended sliding mode observer based control law has been developed. In [17], sliding mode observers are utilized for state and disturbance estimation in electrohydraulic systems. For the vibration control of a train-car suspension with magnetorheological dampers, a SMDO-based controller has been investigated in [18]. For the air-breathing hypersonic flight vehicles subject to external disturbances and actuator saturations, a sliding mode exact disturbance observer (SMEDO) is exploited to exactly estimate the lumped disturbances $[19,20]$. As is stressed in [21], SMDO possess strong robustness and accuracy to estimate a lumped disturbance including unknown external 
disturbances and parametric uncertainties. In [22], a sliding mode disturbance observer with switching-gain adaptation was proposed. In $[23,24]$, the second-order sliding mode disturbance observer, which can produce continuous estimation signals and possess strong robustness, had been proposed and applied to industrial systems.

On the other hand, in the practical control systems, it is of significant importance to consider the transient control performances including the overshoot, undershoot, and convergence rate. However, there are few results focusing on this issue. Usually, the traditional adaptive control systems can only steer the tracking errors to converge to a residual set whose size resting with the control gains and the disturbances ranges $[25,26]$. Recently, by utilizing appropriately defined functions to transform the original system into one that incorporates the desired performance criteria, an effective control scheme which can guarantee the prescribed transient performances has been proposed by Bechlioulis [27]. In [28], a fault-tolerant controller guaranteeing prescribed tracking performance has been proposed for a class of nonlinear uncertain systems. Moreover, the prescribed performance controllers for the cascade systems [29] and the nonaffine nonlinear large scale systems [30] have also been developed.

Since NSVs are not constrained by orbital mechanics and fuel consumption, they can offer significant advantages to Low Earth Orbit (LEO) satellites and airplanes [31]. In general, the NSV has larger flight envelope, rapid flight speed, and time-varying aerodynamic characteristics; the controller design becomes challenging and it is necessary to develop effective control methods to guarantee the safety and reliability [32]. In the past decades, a number of advanced control approaches have been developed for the NSVs. In [33], by introducing the neural networks into an adaptive backstepping controller, an effective attitude controller has been proposed. For the NSV suffering from the dynamical uncertainties, an adaptive functional link network control structure has been constructed in [34]. In [35], a robust attitude controller has been designed for NSVs subjected to time-varying disturbances. In [36], a globally convergent Levenberg-Marquardt (LM) algorithm based on Takagi-Sugeno fuzzy training has been proposed for the NSV. In [37], a terminal sliding mode controller combined with dynamic sliding mode was designed based on nonlinear disturbance observer.
In spite of the progress, the control results for the NSVs with guaranteed transient performances have rarely been reported. Moreover, in practice, the aerodynamic parameters perturbations, measurement errors, actuator faults, and wind effects may induce significant uncertainties in the flight control systems of NSVs. To the best of the authors' knowledge, the existing controllers are commonly designed for the NSVs suffering from only constant disturbances, and the aforementioned quickly varying multiple uncertainties cannot be handled. Therefore, in this paper, we are dedicated to designing an adaptive tracking control structure which can guarantee the prescribed transient tracking performance and possess satisfactory disturbance attention ability. By introducing a second-order sliding mode disturbance observer, the time-varying mismatched uncertainties can be estimated and compensated. With the aid of the hierarchical fuzzy approximator and a novel nonlinear function, the negative effects of the quickly varying multiple matched disturbances can be handled. Based on the performance functions and error transformation, the robustness and predefined tracking performance can be ensured. The adverse actuator derivations are also overcome by the proposed controller. Compared with the previous works, the contributions of this paper are summarized as follows.

(1) An effective adaptive fuzzy control algorithm, which can guarantee the prescribed transient tracking performances including the convergence rate, the tracking error, and the overshoot, is developed for the NSVs.

(2) The NSV model suffering from the multisource quickly varying uncertainties, including the actuator faults, the wind effects, aerodynamic uncertainties, and measurement errors, is established.

(3) The adverse effects of the quickly varying uncertainties can be circumvented. The theoretic developments of this paper are valuable for handling the timevarying disturbances.

\section{Problem Formulation and Preliminaries}

2.1. NSV Model. According to [38, 39], the attitude dynamic model of NSVs can be described by

$$
\begin{aligned}
& \dot{\alpha}=\omega_{z}+\omega_{y} \sin \alpha \tan \beta-\omega_{x} \cos \alpha \tan \beta-\frac{Y+m g \cos \theta \cos \gamma_{v}}{m V \cos \beta} \\
& \dot{\beta}=\omega_{y} \cos \alpha+\omega_{x} \sin \alpha+\frac{Z-m g \cos \theta \sin \gamma_{v}}{m V} \\
& \dot{\gamma}_{v}=\frac{-\omega_{y} \sin \alpha}{\cos \beta}+\frac{\omega_{x} \cos \alpha}{\cos \beta}+\frac{Y\left[\sin \theta \sin \gamma_{v}+\tan \beta\right]+Z \sin \theta \cos \gamma_{v}+m g \cos \beta \cos \gamma_{v} \tan \beta}{m V} \\
& \dot{\omega}_{x}=r_{1} \omega_{x} \omega_{z}+r_{2} \omega_{y} \omega_{z}+r_{3} M_{x}+r_{4} M_{y} \\
& \dot{\omega}_{y}=q_{1} \omega_{x} \omega_{z}+q_{2} \omega_{y} \omega_{z}+r_{4} M_{x}+q_{3} M_{y} \\
& \dot{\omega}_{z}=p_{1} \omega_{x} \omega_{y}+p_{2}\left(\omega_{x}{ }^{2}-\omega_{y}{ }^{2}\right)+p_{3} M_{z}
\end{aligned}
$$


where $\alpha, \beta$, and $\gamma_{v}$ are the angle of attack, sideslip angle, and bank angle of the NSV, respectively. $\theta$ is the flight path angle. $Y$ and $Z$ represent lift and lateral force. $V$ is the velocity of the vehicle. $\omega_{x}, \omega_{y}$, and $\omega_{z}$ are the roll, pitch, and yaw angular rates of the vehicle, respectively. The inertial parameters $p_{1}, p_{2}, p_{3}, q_{1}, q_{2}, q_{3}, r_{1}, r_{2}, r_{3}, r_{4}$ can be formulated by

$$
\begin{aligned}
& p_{1}=\frac{J_{x}-J_{y}}{J_{z}}, \\
& p_{2}=\frac{J_{x y}}{J_{z}}, \\
& p_{3}=\frac{1}{J_{z}}, \\
& q_{1}=\frac{J_{x} J_{z}-J_{x y}^{2}-J_{x}^{2}}{J_{x} J_{y}-J_{x y}^{2}}, \\
& q_{2}=-\frac{J_{x y}\left(J_{x}-J_{y}+J_{z}\right)}{J_{x} J_{y}-J_{x y}^{2}}, \\
& q_{3}=\frac{J_{x}}{J_{x} J_{y}-J_{x y}^{2}}, \\
& r_{4}=\frac{J_{x y}}{J_{x} J_{y}-J_{x y}^{2}} \\
& r_{1}=-\frac{J_{x y}\left(J_{x}+J_{y}-J_{z}\right)}{J_{x} J_{y}-J_{x y}^{2}}, \\
& r_{2}=\frac{J_{y}^{2}-J_{x y}^{2}-J_{y} J_{z}}{J_{x} J_{y}-J_{x y}^{2}},
\end{aligned}
$$

The aerodynamic forces can be described as

$$
\begin{aligned}
& Y=C_{Y} q S \\
& Z=C_{Z} q S
\end{aligned}
$$

where

$$
\begin{aligned}
& C_{Y}=C_{Y}^{\alpha} \alpha+C_{Y}^{\delta_{z}} \delta_{z}+C_{Y}^{\delta_{y}} \delta_{y} \\
& C_{Z}=C_{Z}^{\beta} \beta+C_{Z}^{\delta_{y}} \delta_{y}+C_{Z}^{\delta_{x}} \delta_{x}
\end{aligned}
$$

The aerodynamic moment are as follows:

$$
\begin{aligned}
M_{x} & =q S L\left(m_{x 0}+m_{x}^{\alpha} \alpha+m_{x}^{\beta} \beta+m_{x}^{\delta_{y}} \delta_{y}+m_{x}^{\delta_{x}} \delta_{x}\right. \\
& \left.+m_{x}^{\bar{\omega}_{x}} \bar{\omega}_{x}\right) \\
M_{y} & =q S L\left(m_{y 0}+m_{y}^{\alpha} \alpha+m_{y}^{\beta} \beta+m_{y}^{\delta_{y}} \delta_{y}+m_{y}^{\delta_{x}} \delta_{x}\right. \\
& \left.+m_{y}^{\bar{\omega}_{y}} \bar{\omega}_{y}\right)
\end{aligned}
$$

$$
M_{z}=q S L\left(m_{z 0}+m_{z}^{\alpha} \alpha+m_{z}^{\beta} \beta+m_{z}^{\delta_{z}} \delta_{z}+m_{z}^{\bar{\omega}_{z}} \bar{\omega}_{z}\right)
$$

where $m_{x 0}, m_{y 0}, m_{z 0}$ denote the aerodynamic coefficients when the deflection angles are zero. $m_{x}^{\bar{\omega}_{x}}, m_{y}^{\bar{\omega}_{y}}, m_{y}^{\bar{\omega}_{y}}$ represent the coefficients of aerodynamic damping torque. $C_{Y}$ is the aerodynamic coefficient of the lift force; $C_{Z}$ is the lateral force coefficient. The aerodynamic partial derivatives are represented by $m_{x}^{\alpha}, m_{x}^{\beta}, m_{y}^{\alpha}, m_{y}^{\beta}, m_{z}^{\alpha}, m_{z}^{\beta}, m_{x}^{\delta_{x}}, m_{x}^{\delta_{y}}, m_{y}^{\delta_{x}}, m_{y}^{\delta_{y}}, m_{z}^{\delta_{z}}$. $\delta_{x}, \delta_{y}, \delta_{z}$ are the deflection angles of control surfaces. $S$ is the reference area. $L$ is the reference length. $q$ is dynamic pressure. $\bar{\omega}_{x}, \bar{\omega}_{y}, \bar{\omega}_{z}$ are calculated as

$$
\begin{aligned}
& \bar{\omega}_{x}=\frac{L \omega_{x}}{V}, \\
& \bar{\omega}_{y}=\frac{L \omega_{y}}{V}, \\
& \bar{\omega}_{z}=\frac{L \omega_{z}}{V}
\end{aligned}
$$

It should be highlighted that multiple uncertainties including the aerodynamic uncertainties, measurement errors, and unmodeled dynamics are often encountered in the NSV. Meanwhile, the NSVs often experience a complex flight environment, and the undesired stochastic winds have to be considered. Let $\Delta \alpha$ and $\Delta \beta$ represent the additional angle of attack and the additional sideslip angle, respectively. Then the disturbance forces and torques caused by the unknown winds can be described as

$$
\begin{aligned}
\Delta Y^{\text {Wind }} & =q S C_{Y}^{\alpha} \Delta \alpha, \\
\Delta Z^{\text {Wind }} & =q S C_{Z}^{\beta} \Delta \beta \\
\Delta M_{x}^{\text {Wind }} & =q S L\left(m_{x}^{\alpha} \Delta \alpha+m_{x}^{\beta} \Delta \beta\right) \\
\Delta M_{y}^{\text {Wind }} & =q S L\left(m_{y}^{\alpha} \Delta \alpha+m_{y}^{\beta} \Delta \beta\right) \\
\Delta M_{z}^{\text {Wind }} & =q S L\left(m_{z}^{\alpha} \Delta \alpha+m_{z}^{\beta} \Delta \beta\right)
\end{aligned}
$$

Moreover, the aerodynamic-perturbation-caused disturbance forces and torques are defined as $\Delta Y^{P t b}, \Delta Z^{P t b}, \Delta M_{x}^{P t b}$, $\Delta M_{y}^{P t b}, \Delta M_{z}^{P t b}$.

In general, the NSVs are commonly equipped with the electromechanical aerodynamic rudders. However, there often exist multifarious faults in the aerodynamic rudders. As is revealed in $[3,4]$, actuator faults constitute the main reason for the undesired control performance and instability of the flight control system. Define $\boldsymbol{u}=\left[\begin{array}{lll}\delta_{z} & \delta_{y} & \delta_{x}\end{array}\right]^{T}$. The faulty actuator model can be provided as

$$
\boldsymbol{u}_{c}=\boldsymbol{u}+\Delta \boldsymbol{\delta}
$$

where $\boldsymbol{u}$ and $\boldsymbol{u}_{c}$ denote the input and output of the actuator, respectively. $\Delta \boldsymbol{\delta}$ denotes the deviation caused by the actuator faults. It is supposed that $|\Delta \delta| \leq \Delta \bar{\delta}$.

The neglect of the above-mentioned multiple uncertainties and actuator faults may cause worse control performance and instability of the flight control system. The intrinsic high 
dynamic characteristics and the strong coupled properties make it a challenge to establish an effective control scheme. Define $\boldsymbol{x}_{1}=\left[\begin{array}{lll}\alpha & \beta & \gamma_{v}\end{array}\right]^{T}$ and $\boldsymbol{x}_{2}=\left[\begin{array}{lll}\omega_{z} & \omega_{y} & \omega_{x}\end{array}\right]^{T}$. Taking the multiple uncertainties into consideration, we can get the following NSV model:

$$
\begin{aligned}
& \dot{x}_{1}=A x_{2}+f_{1}\left(x_{1}, x_{2}\right)+\Delta f_{1}\left(x_{1}, x_{2}\right)+D_{F} \\
& \dot{x}_{2}=f_{2}\left(x_{1}, x_{2}\right)+\Delta f_{2}\left(x_{1}, x_{2}\right)+B u+B \Delta \delta+D_{M}
\end{aligned}
$$

where

$$
\begin{aligned}
& \mathbf{A}=\left[\begin{array}{ccc}
1 & \sin \alpha \tan \beta & -\cos \alpha \tan \beta \\
0 & \cos \alpha & \sin \alpha \\
0 & -\frac{\sin \alpha}{\cos \beta} & \frac{\cos \alpha}{\cos \beta}
\end{array}\right] \\
& \mathbf{B}=q S L\left[\begin{array}{ccc}
p_{3} & 0 & 0 \\
0 & q_{3} & r_{4} \\
0 & r_{4} & r_{3}
\end{array}\right]\left[\begin{array}{ccc}
m_{z}^{\delta_{z}} & 0 & 0 \\
0 & m_{y}^{\delta_{y}} & m_{y}^{\delta_{x}} \\
0 & m_{x}^{\delta_{y}} & m_{x}^{\delta_{x}}
\end{array}\right] \\
& \mathbf{f}_{1}\left(\mathbf{x}_{1}, \mathbf{x}_{2}\right)=\left[\begin{array}{c}
-\frac{Y+m g \cos \theta \cos \gamma_{v}}{m V \cos \beta} \\
\frac{Z-m g \cos \theta \sin \gamma_{v}}{m V} \\
\frac{Y\left[\sin \theta \sin \gamma_{v}+\tan \beta\right]+Z \sin \theta \cos \gamma_{v}+m g \cos \theta \cos \gamma_{v} \tan \beta}{m V}
\end{array}\right] \\
& \mathbf{f}_{2}\left(\mathbf{x}_{1}, \mathbf{x}_{2}\right)=\left[\begin{array}{c}
p_{1} \omega_{x} \omega_{y}+p_{2}\left(\omega_{x}{ }^{2}-\omega_{y}{ }^{2}\right) \\
q_{1} \omega_{x} \omega_{z}+q_{2} \omega_{y} \omega_{z} \\
r_{1} \omega_{x} \omega_{z}+r_{2} \omega_{y} \omega_{z}
\end{array}\right]+q S L\left(\left[\begin{array}{ccc}
p_{3} & 0 & 0 \\
0 & q_{3} & r_{4} \\
0 & r_{4} & r_{3}
\end{array}\right]\left[\begin{array}{c}
m_{z 0} \\
m_{y 0} \\
m_{x 0}
\end{array}\right]+\left[\begin{array}{ccc}
p_{3} & 0 & 0 \\
0 & q_{3} & r_{4} \\
0 & r_{4} & r_{3}
\end{array}\right]\left[\begin{array}{c}
C_{m q} \bar{\omega}_{z} \\
C_{n r} \bar{\omega}_{y} \\
C_{l p} \bar{\omega}_{x}
\end{array}\right]\right) \\
& \mathbf{D}_{F}=\mathbf{D}_{F, 0}+\mathbf{D}_{F, 1} \\
& \mathbf{D}_{F, 0}=\left[\begin{array}{c}
-\frac{\Delta Y}{m V \cos \beta} \\
\frac{\Delta Z}{m V} \\
\frac{\Delta Y\left[\sin \theta \sin \gamma_{v}+\tan \beta\right]+\Delta Z \sin \theta \cos \gamma_{v}}{m V}
\end{array}\right] \\
& \mathbf{D}_{M}=q S L\left[\begin{array}{ccc}
p_{3} & 0 & 0 \\
0 & q_{3} & r_{4} \\
0 & r_{4} & r_{3}
\end{array}\right]\left[\begin{array}{l}
\Delta M_{x}^{P t b}+\Delta M_{x}^{\text {Wind }}+\Delta M_{x}^{\text {Ass }}+\Delta M_{x}^{F a b} \\
\Delta M_{y}^{P t b}+\Delta M_{y}^{\text {Wind }}+\Delta M_{y}^{\text {Ass }}+\Delta M_{y}^{F a b} \\
\Delta M_{z}^{P t b}+\Delta M_{z}^{\text {Wind }}+\Delta M_{z}^{\text {Ass }}+\Delta M_{z}^{F a b}
\end{array}\right]+\Delta \mathbf{B u} \\
& \Delta Y=\Delta Y^{P t b}+\Delta Y^{W i n d} \\
& \Delta Z=\Delta Z^{P t b}+\Delta Z^{\text {Wind }}
\end{aligned}
$$

$\Delta \mathbf{f}_{1}\left(\mathbf{x}_{1}, \mathbf{x}_{2}\right)$ and $\Delta \mathbf{f}_{2}\left(\mathbf{x}_{1}, \mathbf{x}_{2}\right)$ are the unknown nonlinearities caused by the model simplification. $\mathbf{D}_{F, 1}$ denotes the disturbances caused by inaccurate measurement information. $\Delta \mathbf{B}$ represents the uncertainty part of the control distribution matrix, which is given as

$$
\Delta \mathbf{B}=q S L\left[\begin{array}{ccc}
p_{3} & 0 & 0 \\
0 & q_{3} & r_{4} \\
0 & r_{4} & r_{3}
\end{array}\right]\left[\begin{array}{ccc}
\Delta m_{z}^{\delta_{z}} & 0 & 0 \\
0 & \Delta m_{y}^{\delta_{y}} & \Delta m_{y}^{\delta_{x}} \\
0 & \Delta m_{x}^{\delta_{y}} & \Delta m_{x}^{\delta_{x}}
\end{array}\right]
$$

In practice, the transient performance including the convergence rate, the tracking error, and the predefined maximum overshoot should be guaranteed. Therefore, the design objective of this work is to establish an effective control structure to force the angle of attack $\alpha$, the sideslip angle $\beta$, and the bank angle $\gamma_{V}$ to track the reference trajectories $\alpha_{r}, \beta_{r}, \gamma_{V, r}$ with predefined transient performance in the simultaneous presence of the actuator faults and multiple uncertainties.

2.2. Assumptions and Supporting Lemmas. The following assumptions and lemmas are necessary in this work.

Assumption 1. The actuator deviations are supposed to satisfy $|\Delta \boldsymbol{\delta}| \leq \Delta \bar{\delta}$. 
Assumption 2. The disturbances existing in the NSV system are assumed to be bounded; i.e., $\left\|\Delta \mathbf{f}_{1}\left(\mathbf{x}_{1}, \mathbf{x}_{2}\right)\right\| \leq \bar{d}_{f},\left\|\boldsymbol{D}_{F}\right\| \leq$ $\bar{d}_{D_{F}},\left\|\boldsymbol{D}_{M}\right\| \leq \bar{d}_{D_{M}}$.

Lemma 3. Consider the system

$$
\begin{aligned}
\dot{x} & =f(x), \\
f(0) & =0, \\
x & \in \mathbb{R}^{n}, \\
x(0) & =x_{0}
\end{aligned}
$$

where $f: D \longrightarrow \mathbb{R}^{n}$ is continuous on an open neighborhood $D$ and the origin is 0. Suppose there is a continuous function $V(x): D \longrightarrow \mathbb{R}^{n}$ defined on $U \subseteq D$ with the origin 0 such that the following conditions hold:

(1) $V(x)$ is positive definite on $D \subseteq \mathbb{R}^{n}$.

(2) There exist real numbers $k>0$ and $v \in(0,1)$, such that $\dot{V}(x)+k V^{v}(x) \leq 0$, and then, system (12) is locally finite-time stable. The settling time, depending on the initial state $x(0)=$ $x_{0}$, satisfies

$$
T\left(x_{0}\right) \leq \frac{V\left(x_{0}\right)^{1-v}}{k(1-v)}
$$

for all $x_{0}$ in some open neighborhood of the origin. If $D=\mathbb{R}^{n}$ and $V(x)$ is also unbounded, system (12) is globally finite-time stable.

Lemma 4 (see [26]). Given any $\varepsilon>0$ and $z \in \mathbb{R}^{n}$, then one has

$$
0 \leq\|z\|-\frac{z^{T} z}{\sqrt{z^{T} z+\varepsilon^{2}}}<\varepsilon
$$

2.3. Hierarchical Fuzzy Systems. For the purpose of compensating the unknown nonlinear functions, we introduce a HFS in the controller. Commonly, the HFS contains several low dimensional fuzzy systems. The structure of the HFS is provided in Figure 1. Let $\boldsymbol{x}=\left[x_{1}, x_{2}, \cdots, x_{n}\right]^{T}$ represent the input of the HFS. Then the first subsystem can be established by using the following rules.

Rule $^{l}$. If $x_{1}$ is $F_{1}^{l}$ and $x_{2}$ is $F_{2}^{l}$, then $y_{1}$ is $G_{1}^{l}, l=1,2, \ldots, N_{1}$.

$N_{1}$ is the total number of fuzzy rules for the first fuzzy subsystem; $F_{1}^{l}, F_{2}^{l}, G_{1}^{l}$ are the corresponding fuzzy sets , $\mu_{F_{k}^{l}}\left(x_{k}\right), k=1,2$, and $\mu_{G^{l}}\left(y_{1}\right)$ represent the fuzzy functions. A subsystem includes a singleton fuzzifier, a center-average defuzzifier, and a product inference engine. The following equation provides the output of a fuzzy subsystem:

$$
\begin{aligned}
y_{1}\left(x_{1}, x_{2}\right) & =\frac{\sum_{l=1}^{N_{1}} w_{1, l} \mu_{F_{1}^{l}}\left(x_{1}\right) \mu_{F_{2}^{l}}\left(x_{2}\right)}{\sum_{l=1}^{N_{1}} \mu_{F_{1}^{l}}\left(x_{1}\right) \mu_{F_{2}^{l}}\left(x_{2}\right)} \\
& =W_{1}^{T} \Phi_{1}\left(x_{1}, x_{2}\right)
\end{aligned}
$$

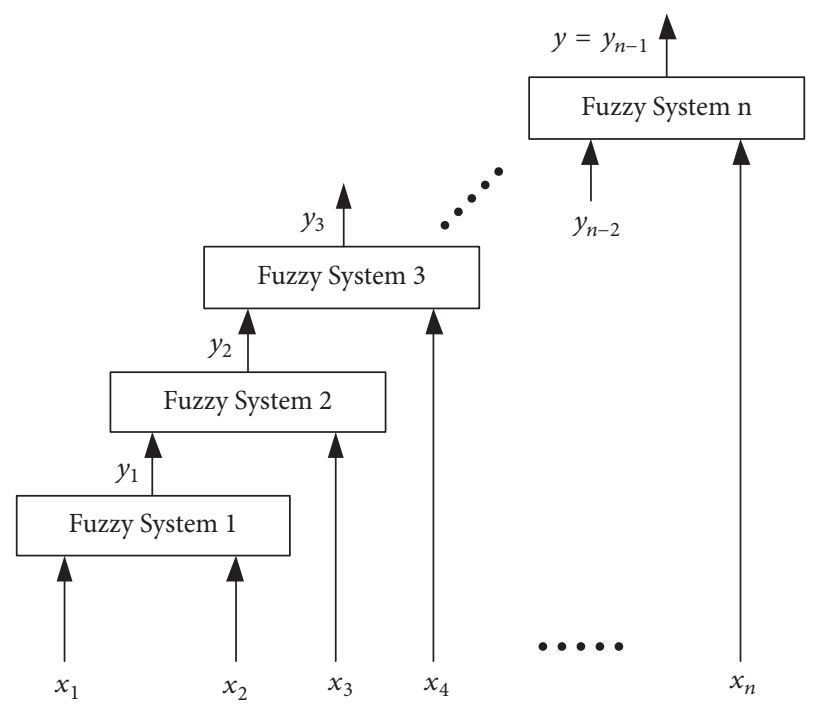

FIGURE 1: The structure of the hierarchical fuzzy system.

where $W_{1}=\left[w_{1,1}, \cdots, w_{1, N_{1}}\right]^{T}$.

$$
\begin{aligned}
\Phi_{1}\left(x_{1}, x_{2}\right) & =\left[\Phi_{1,1}\left(x_{1}, x_{2}\right), \cdots, \Phi_{1, N_{1}}\left(x_{1}, x_{2}\right)\right]^{T} \\
\Phi_{1, l}\left(x_{1}, x_{2}\right) & =\frac{\mu_{F_{1}^{l}}\left(x_{1}\right) \mu_{F_{2}^{l}}\left(x_{2}\right)}{\sum_{l=1}^{N_{1}} \mu_{F_{1}^{l}}\left(x_{1}\right) \mu_{F_{2}^{l}}\left(x_{2}\right)}
\end{aligned}
$$

Then, we can establish the $i$-th $(i=2, \cdots, n-1)$ fuzzy subsystem with the aid of the following rules.

Rule $e^{l}$. If $x_{i+1}$ is $F_{i+1}^{l}$ and $y_{i-1}$ is $G_{i-1}^{l}$, then $y_{i}$ is $G_{i}^{l}, l=1,2, \ldots, N_{i}$.

$x_{i+1}$ and $y_{i-1}$ are the inputs, and $y_{i}$ is the output. Hence, $y_{i}$, which is the output of the $i$-th fuzzy subsystem, is calculated as

$$
\begin{aligned}
y_{i} & =\frac{\sum_{l=1}^{N_{i}} w_{i, l} \mu_{G_{i-1}^{l}}\left(y_{i-1}\right) \mu_{F_{i+1}^{l}}\left(x_{i+1}\right)}{\sum_{l=1}^{N_{i}} \mu_{G_{i-1}^{l}}\left(y_{i-1}\right) \mu_{F_{i+1}^{l}}\left(x_{i+1}\right)} \\
& =W_{i}^{T} \Phi_{i}\left(y_{i-1}, x_{i+1}\right)
\end{aligned}
$$

Finally, a hierarchical fuzzy system can be established and the final output can be given as

$$
y_{n-1}=W_{n-1}^{T} \Phi_{n-1}\left(y_{n-2}, x_{n}\right)
$$

It is clear that the total number of fuzzy rules is $\prod_{i=1}^{n} N_{i}^{2}$.

Lemma 5 (see [40]). Define $U=\left[a_{1}, b_{1}\right] \times \cdots \times\left[a_{n}, b_{n}\right]$. Consider a nonlinear function $f\left(x_{1}, \cdots, x_{n}\right)$, which is continuously differentiable and defined on $U$. Then we can construct a hierarchical fuzzy system $y\left(x_{1}, \cdots, x_{n}\right)$ within (15) (18) such that

$$
\|f(\boldsymbol{x})-y(\boldsymbol{x})\|_{\infty} \leq \sum_{i=1}^{n}\left(\left\|\frac{\partial f}{\partial x_{i}}\right\|_{\infty}+\left\|\frac{\partial y}{\partial x_{i}}\right\|_{\infty}\right) \frac{b-a}{N_{i}-1}
$$

where $\boldsymbol{x}=\left[x_{1}, \cdots, x_{n}\right]^{T}$. 
Lemma 6 (see [40]). Define $U=\left[a_{1}, b_{1}\right] \times \cdots \times\left[a_{n}, b_{n}\right]$. Consider any function $f\left(x_{1}, \cdots, x_{n}\right)$ which is continuously differentiable and defined on $U$. Then one can construct a hierarchical fuzzy system $y\left(x_{1}, \cdots, x_{n}\right)$ within $(15) \sim(18)$ such that $\sup _{x \in U}|f(\boldsymbol{x})-y(\boldsymbol{x})|<\varepsilon$, where $\varepsilon$ is an arbitrarily small positive constant.

\section{Main Results}

3.1. Performance Functions. Define $e_{\alpha}=\alpha-\alpha_{r}, e_{\beta}=\beta-\beta_{r}$ and $e_{\gamma_{V}}=\gamma_{V}-\gamma_{V, r}$. Then, for the purpose of guaranteeing the predefined control performance, we introduce the performance functions in the following text. Define the following exponentially decaying functions $p_{i}(t), i \in\left\{\alpha, \beta, \gamma_{V}\right\}$ as the performance functions:

$$
\begin{aligned}
& p_{i}(t)=\left(p_{i, 0}-p_{i, \infty}\right) \exp \left(-a_{i} t\right)+p_{i, \infty}, \\
& i \in\left\{\alpha, \beta, \gamma_{V}\right\}
\end{aligned}
$$

where $p_{i, 0}>p_{i, \infty}>0$ and $a_{i}>0$. Define $\underline{b}_{i}, \bar{b}_{i}>0$. If the inequality $-\underline{b}_{i} p_{i}(t)<e_{i}(t)<\bar{b}_{i} p_{i}(t)$ holds for $t \geq 0$, then the transient performance is ensured. To make this point more clear, we make the following explanations. $\bar{b}_{i} p_{i, 0}$ and $-\underline{b}_{i} p_{i, 0}$ restrict the range of the overshoot and the lower bound of the undershoot of $e_{i}(t)$. The convergence speed of $e_{i}(t)$ is restrained by the decreasing speed of $p_{i}(t)$. The steady error $e_{i}(t)$ is constrained by $\max \left\{\underline{b}_{i} p_{i, \infty}, \bar{b}_{i} p_{i, \infty}\right\}$.

Consider the following strictly increasing function:

$$
\begin{aligned}
s_{i}=T\left(\frac{e_{i}}{p_{i}}\right)=\ln \left(\underline{b}_{i}+\frac{e_{i}}{p_{i}}\right)-\ln \left(\bar{b}_{i}-\frac{e_{i}}{p_{i}}\right), & \\
& i \in\left\{\alpha, \beta, \gamma_{V}\right\}
\end{aligned}
$$

Clearly, function (21) satisfies

$$
\begin{aligned}
\lim _{e_{i} / p_{i} \longrightarrow-\underline{b}_{i}} T\left(\frac{e_{i}}{p_{i}}\right) & =-\infty, \\
\lim _{e_{i} / p_{i} \longrightarrow \bar{b}_{i}} T\left(\frac{e_{i}}{p_{i}}\right) & =+\infty
\end{aligned}
$$

It is not difficult to verify that the inverse function $S^{-1}(\bullet)$ is well-defined and strictly increasing as well. Obviously, if $s_{i}$ is bounded, $e_{i}(t) / p_{i}(t)$ remains within a compact subset of $\left(-\underline{b}_{i}, \bar{b}_{i}\right)$. As a result, the control issue forces $s_{i}$ to be bounded.

\subsection{Second-Order Sliding Mode Disturbance Observer.}

Define $\boldsymbol{x}_{1, r}=\left[\alpha_{r}, \beta_{r}, \gamma_{V, r}\right]^{T}$ and $\boldsymbol{e}_{1}=\boldsymbol{x}_{1}-\boldsymbol{x}_{1, r}$. Consider the NSV model given in (9); we can get the following dynamic equation:

$$
\begin{aligned}
& \dot{e}_{1}=A x_{2}+f_{1}\left(x_{1}, x_{2}\right)+\Delta f_{1}\left(x_{1}, x_{2}\right)+D_{F}-x_{1, r} \\
& \dot{x}_{2}=f_{2}\left(x_{1}, x_{2}\right)+\Delta f_{2}\left(x_{1}, x_{2}\right)+B u+B \Delta \delta+D_{M}
\end{aligned}
$$

Using the strictly increasing function provided in (21), it can be proven that

$$
\begin{aligned}
\dot{\boldsymbol{s}} & =\overline{\boldsymbol{s}}\left(A x_{2}+f_{1}\left(x_{1}, x_{2}\right)+\Delta f_{1}\left(x_{1}, x_{2}\right)+D_{F}-x_{1, r}\right. \\
& -\psi) \\
\dot{x}_{2} & =f_{2}\left(x_{1}, x_{2}\right)+\Delta f_{2}\left(x_{1}, x_{2}\right)+B u+B \Delta \delta+D_{M}
\end{aligned}
$$

where

$$
\begin{aligned}
\boldsymbol{s} & =\left[s_{\alpha}, s_{\beta}, s_{\gamma_{V}}\right]^{T}, \\
\overline{\boldsymbol{s}} & =\operatorname{diag}\left[\bar{s}_{\alpha}, \bar{s}_{\beta}, \bar{s}_{\gamma_{V}}\right] \\
\psi & =\left[\psi_{\alpha}, \psi_{\beta}, \psi_{\gamma_{V}}\right]^{T} \\
\bar{s}_{i} & =\frac{\partial T}{\partial\left(e_{i} / p_{i}\right)} \frac{1}{p_{i}}, \\
\psi_{i} & =\frac{e_{i} \dot{p}_{i}}{p_{i}},
\end{aligned}
$$

$$
i \in\left\{\alpha, \beta, \gamma_{V}\right\}
$$

Consider the mismatched uncertainties $\boldsymbol{d}_{\text {mis }}=\Delta \boldsymbol{f}_{1}\left(\boldsymbol{x}_{1}, \boldsymbol{x}_{2}\right)+$ $D_{F}$ existing in system, a second-order sliding mode disturbance observer is introduced for estimation and compensation. The SOSMDO is designed as

$$
\begin{aligned}
\widehat{\boldsymbol{d}}_{\text {mis }} & =\mathbf{v}, \\
\mathbf{v} & =-k_{1} \frac{\boldsymbol{\xi}}{\|\boldsymbol{\xi}\|^{1 / 2}}+\boldsymbol{z}-k_{2} \boldsymbol{\xi}, \\
\dot{\boldsymbol{z}} & =-k_{3} \frac{\boldsymbol{\xi}}{\|\boldsymbol{\xi}\|}-k_{4} \boldsymbol{\xi} \\
\boldsymbol{\xi} & =\boldsymbol{z}_{0}-\boldsymbol{e}_{1}, \\
\dot{\boldsymbol{z}}_{0} & =\boldsymbol{A} \boldsymbol{x}_{2}+\boldsymbol{f}_{1}\left(\boldsymbol{x}_{1}, \boldsymbol{x}_{2}\right)-\boldsymbol{x}_{1, \boldsymbol{r}}+\mathbf{v}
\end{aligned}
$$

In this work, we divide the mismatched uncertainties into two parts. One is the unknown disturbance related to the system states; the other is the time-varying unknown uncertainty. In other words,

$$
\boldsymbol{d}_{\text {mis }}=\boldsymbol{d}_{1}(t, \boldsymbol{\xi})+\int_{0}^{t} \boldsymbol{d}_{2}(\tau) d \tau
$$

Without loss of generality, it is supposed that there exist constants $g_{1}, g_{2}$ such that

$$
\begin{gathered}
\left\|\boldsymbol{d}_{1}(t, \boldsymbol{\xi})\right\| \leq g_{1}\|\boldsymbol{\xi}\|, \\
\left\|\boldsymbol{d}_{2}\right\| \leq g_{2}
\end{gathered}
$$

Theorem 7. Consider system (23). If one selects $k_{1}, \cdots, k_{4}$ such that

$$
\begin{aligned}
& k_{1}>\sqrt{2 g_{2}}, \\
& k_{2}>2 g_{1}, \\
& k_{3}>\max \left(\bar{k}_{3}^{\Theta}, \bar{k}_{3}^{\Upsilon}\right), \\
& k_{4}>\max \left(\bar{k}_{4}^{\Theta}, \bar{k}_{4}^{\Upsilon}\right),
\end{aligned}
$$


where

$$
\begin{aligned}
\bar{k}_{3}^{\Theta} & =3 g_{2}+\frac{2 g_{2}^{2}}{k_{1}^{2}}, \\
\bar{k}_{4}^{\Theta} & =\frac{\left((3 / 2) k_{1}^{2} k_{2}+3 g_{2} k_{2}\right)^{2}}{k_{3} k_{1}^{2}-2 g_{2}^{2}-3 g_{2} k_{1}^{2}}+k_{2}^{2}+\frac{3}{2} k_{2} g_{1} \\
\bar{k}_{3} & =\frac{(9 / 16)\left(k_{1} g_{1}\right)^{2}}{k_{2}\left(k_{2}-2 g_{1}\right)}+\frac{(1 / 2) k_{1}^{2} g_{1}-2 k_{1}^{2} k_{2}+k_{2} g_{2}}{\left(k_{2}-2 g_{1}\right)} \\
\bar{k}_{4}^{\Upsilon} & =\frac{1}{\left(k_{2}-2 g_{1}\right)} \\
& \cdot \frac{(9 / 16)\left(k_{1} g_{1}\right)^{2}\left(k_{2}+(1 / 2) g_{1}\right)^{2} / k_{2}^{2}}{k_{2}\left(k_{3}+2 k_{1}^{2}-g_{2}\right)-\left(2 k_{3}+(1 / 2) k_{1}^{2}\right) g_{1}-(9 / 16)\left(k_{1} g_{1}\right)^{2}} \\
& +\frac{2 k_{2}^{2} g_{1}+(1 / 4) k_{2} g_{1}^{2}}{\left(k_{2}-2 g_{1}\right)}
\end{aligned}
$$

then the estimation error of the mismatched uncertainties can be forced to zero in finite time.

Proof. From (23) and (26), we can get the following closedloop dynamic equation:

$$
\begin{aligned}
& \dot{\xi}=-k_{1} \frac{\xi}{\|\xi\|^{1 / 2}}+\boldsymbol{z}-k_{2} \boldsymbol{\xi}-\boldsymbol{d}_{\text {mis }} \\
& \dot{z}=-k_{3} \frac{\xi}{\|\xi\|}-k_{4} \xi
\end{aligned}
$$

Consider the following Lyapunov function:

$$
V(\boldsymbol{\xi}, \boldsymbol{z})=2 k_{3}\|\boldsymbol{\xi}\|+k_{4} \xi^{T} \boldsymbol{\xi}+\frac{1}{2} \boldsymbol{z}^{T} \boldsymbol{z}+\boldsymbol{\eta}^{T} \boldsymbol{\eta}
$$

where $\boldsymbol{\eta}=k_{1} \xi\|\xi\|^{-1 / 2}+k_{2} \xi-z$. Taking the derivatives of (32) yields

$$
\begin{aligned}
\dot{V}(\boldsymbol{\xi}, \boldsymbol{z})= & \left(2 k_{3}+\frac{k_{1}{ }^{2}}{2}\right) \frac{\boldsymbol{\xi}^{T} \dot{\boldsymbol{\xi}}}{\|\boldsymbol{\xi}\|}+2\left(\frac{k_{1}^{2}}{2}+k_{4}\right) \boldsymbol{\xi}^{T} \dot{\boldsymbol{\xi}} \\
& +2 \boldsymbol{z}^{T} \dot{\boldsymbol{z}}+\frac{3}{2} k_{1} k_{2} \frac{\xi^{T} \dot{\boldsymbol{\xi}}}{\|\boldsymbol{\xi}\|^{1 / 2}}-k_{2}\left(\dot{\boldsymbol{\xi}}^{T} \boldsymbol{z}+\boldsymbol{\xi}^{T} \dot{\boldsymbol{z}}\right) \\
& -k_{1}\left(-\frac{1}{2} \frac{\left(\xi^{T} \dot{\boldsymbol{\xi}}\right)\left(\boldsymbol{z}^{T} \boldsymbol{\xi}\right)}{\|\boldsymbol{\xi}\|^{5 / 2}}+\frac{\left(\dot{\boldsymbol{z}}^{T} \boldsymbol{\xi}\right)\left(\boldsymbol{z}^{T} \dot{\boldsymbol{\xi}}\right)}{\|\boldsymbol{\xi}\|^{1 / 2}}\right)
\end{aligned}
$$

Substituting (31) into (33) yields

$$
\begin{aligned}
\dot{V}(\boldsymbol{\xi}, \boldsymbol{z})= & -\left(k_{1} k_{3}+\frac{k_{1}{ }^{3}}{2}\right) \frac{\|\boldsymbol{\xi}\|^{2}}{\|\boldsymbol{\xi}\|^{3 / 2}}+\frac{3}{2} k_{1} k_{2} \frac{\boldsymbol{\xi}^{T} \boldsymbol{d}_{1}}{\|\boldsymbol{\xi}\|^{1 / 2}} \\
& -\left(k_{2} k_{4}+k_{2}{ }^{3}\right)\|\boldsymbol{\xi}\|^{2} \\
& -\left(k_{4} k_{1}+\frac{5}{2} k_{1} k_{2}{ }^{2}\right) \frac{\|\boldsymbol{\xi}\|^{2}}{\|\boldsymbol{\xi}\|^{1 / 2}}+k_{1}{ }^{2} \frac{\boldsymbol{\xi}^{T} \boldsymbol{z}}{\|\boldsymbol{\xi}\|} \\
& +2 k_{2}{ }^{2} \boldsymbol{\xi}^{T} \boldsymbol{z}+3 k_{1} k_{2} \frac{\boldsymbol{\xi}^{T} \boldsymbol{z}}{\|\boldsymbol{\xi}\|^{1 / 2}}-k_{2}\|\boldsymbol{z}\|^{2}
\end{aligned}
$$

$$
\begin{aligned}
& +\frac{k_{1}}{2} \frac{\left(\boldsymbol{\xi}^{T} \boldsymbol{z}\right)\left(\boldsymbol{z}^{T} \boldsymbol{\xi}\right)}{\|\xi\|^{5 / 2}}-k_{1} \frac{\boldsymbol{z}^{T} \boldsymbol{z}}{\|\boldsymbol{\xi}\|^{1 / 2}} \\
& +\left(2 k_{3}+\frac{k_{1}^{2}}{2}\right) \frac{\boldsymbol{\xi}^{T} \boldsymbol{d}_{1}}{\|\boldsymbol{\xi}\|}+\left(2 k_{4}+k_{2}^{2}\right) \boldsymbol{\xi}^{T} \boldsymbol{d}_{1} \\
& -\left(k_{3} k_{2}+2 k_{1}^{2} k_{2}\right) \frac{\|\boldsymbol{\xi}\|^{2}}{\|\boldsymbol{\xi}\|}-k_{2} \boldsymbol{d}_{1}^{T} \boldsymbol{z} \\
& +\frac{k_{1}}{2} \frac{\boldsymbol{\xi}^{T} \boldsymbol{d}_{1} \boldsymbol{z}^{T} \boldsymbol{\xi}}{\|\boldsymbol{\xi}\|^{5 / 2}}-k_{1} \frac{\boldsymbol{z}^{T} \boldsymbol{d}_{1}}{\|\boldsymbol{\xi}\|^{1 / 2}}+2 \boldsymbol{z}^{T} \boldsymbol{d}_{2} \\
& -k_{2} \boldsymbol{\xi}^{T} \boldsymbol{d}_{2}-k_{1} \frac{\boldsymbol{d}_{2}^{T} \boldsymbol{\xi}}{\|\boldsymbol{\xi}\|^{1 / 2}}
\end{aligned}
$$

Furthermore,

$$
\begin{aligned}
& \dot{V}(\xi, z) \leq-\left(k_{1} k_{3}+\frac{k_{1}^{3}}{2}\right)\|\xi\|^{1 / 2} \\
& -\left(k_{3} k_{2}+2 k_{1}{ }^{2} k_{2}\right)\|\xi\|-\left(k_{2} k_{4}+k_{2}^{3}\right)\|\xi\|^{2} \\
& -\left(k_{4} k_{1}+\frac{5}{2} k_{1} k_{2}{ }^{2}\right)\|\xi\|^{3 / 2}+k_{1}{ }^{2} \frac{\left|\xi^{T} z\right|}{\|\xi\|} \\
& +2 k_{2}{ }^{2}\left|\xi^{T} z\right|+3 k_{1} k_{2} \frac{\xi^{T} z}{\|\xi\|^{1 / 2}}-k_{2}\|z\|^{2} \\
& +\frac{k_{1}}{2} \frac{\left|\xi^{T} z\right|^{2}}{\|\boldsymbol{\xi}\|^{5 / 2}}+\left(2 k_{3}+\frac{k_{1}^{2}}{2}\right) \frac{\left|\boldsymbol{\xi}^{T} \boldsymbol{d}_{1}\right|}{\|\boldsymbol{\xi}\|} \\
& +\left(2 k_{4}+k_{2}^{2}\right)\left|\xi^{T} \boldsymbol{d}_{1}\right|+\frac{3}{2} k_{1} k_{2} \frac{\left|\boldsymbol{\xi}^{T} \boldsymbol{d}_{1}\right|}{\|\boldsymbol{\xi}\|^{1 / 2}} \\
& +k_{2}\left|\boldsymbol{d}_{1}^{T} \boldsymbol{z}\right|+\frac{k_{1}}{2} \frac{\left|\xi^{T} \boldsymbol{d}_{1}\right|\left|\boldsymbol{z}^{T} \xi\right|}{\|\xi\|^{5 / 2}}+k_{1} \frac{\left|\boldsymbol{z}^{T} \boldsymbol{d}_{1}\right|}{\|\boldsymbol{\xi}\|^{1 / 2}} \\
& +2 \boldsymbol{z}^{T} \boldsymbol{d}_{2}+k_{2}\left|\boldsymbol{\xi}^{T} \boldsymbol{d}_{2}\right|+k_{1} \frac{\left|\boldsymbol{d}_{2}^{T} \boldsymbol{\xi}\right|}{\|\boldsymbol{\xi}\|^{1 / 2}}
\end{aligned}
$$

By using (28) we can get that

$$
\begin{aligned}
\dot{V}(\xi, z) \leq & -\left(k_{1} k_{3}+\frac{k_{1}^{3}}{2}\right)\|\xi\|^{1 / 2} \\
& -\left(k_{3} k_{2}+2{k_{1}}^{2} k_{2}\right)\|\xi\| \\
& -\left(k_{4} k_{1}+\frac{5}{2} k_{1} k_{2}^{2}\right)\|\xi\|^{3 / 2}+k_{1}^{2}\|z\| \\
& -\left(k_{2} k_{4}+k_{2}^{3}\right)\|\xi\|^{2}+2 k_{2}^{2}\|\xi\|\|z\| \\
& +3 k_{1} k_{2}\|\xi\|^{1 / 2}\|z\|-k_{2}\|z\|^{2}+\frac{k_{1}}{2} \frac{\|z\|^{2}}{\|\xi\|^{1 / 2}} \\
& +\left(2 k_{3}+\frac{k_{1}^{2}}{2}\right) g_{1}\|\xi\|
\end{aligned}
$$




$$
\begin{aligned}
& +\left(2 k_{4}+k_{2}^{2}\right) g_{1}\|\xi\|^{2}+\frac{3}{2} k_{1} k_{2}\|\xi\|^{3 / 2} g_{1} \\
& +k_{2} g_{1}\|\xi\|\|z\|+\frac{3}{2} k_{1} g_{1}\|\xi\|^{1 / 2}\|z\| \\
& +2 g_{2}\|z\|+k_{2} g_{2}\|\xi\|+k_{1} g_{2}\|\xi\|^{1 / 2}
\end{aligned}
$$

Denote $\zeta=\operatorname{col}\left(\|\xi\|^{1 / 2},\|\xi\|,\|z\|\right)$; then we can rewrite (36) as

$$
\dot{V} \leq-\frac{1}{\|\xi\|^{1 / 2}} \zeta^{T} \Theta \zeta-\zeta^{T} \Upsilon \zeta
$$

where

$$
\begin{aligned}
& \Theta=\left[\begin{array}{ccc}
\Theta_{11} & 0 & \Theta_{13} \\
0 & \Theta_{22} & \Theta_{23} \\
\Theta_{31} & \Theta_{32} & \Theta_{33}
\end{array}\right], \\
& \Upsilon=\left[\begin{array}{ccc}
\Upsilon_{11} & 0 & \Upsilon_{13} \\
0 & \Upsilon_{22} & \Upsilon_{23} \\
\Upsilon_{31} & \Upsilon_{32} & \Upsilon_{33}
\end{array}\right], \\
& \Theta_{11}=\frac{1}{2} k_{1}^{3}+k_{1} k_{3}-g_{2} k_{1} \text {, } \\
& \Theta_{13}=-\frac{1}{2} k_{1}^{2}-g_{2} \text {, } \\
& \Theta_{22}=k_{4} k_{1}+\frac{5}{2} k_{2}^{2} k_{1}-\frac{3}{2} k_{1} k_{2} g_{1} \text {, } \\
& \Theta_{23}=-\frac{3}{2} k_{1} k_{2}, \\
& \Theta_{31}=\Theta_{13} \text {, } \\
& \Theta_{32}=\Theta_{23} \text {, } \\
& \Theta_{33}=\frac{1}{2} k_{1}, \\
& \Upsilon_{11}=k_{2} k_{3}+2 k_{1}^{2} k_{2}-k_{2} g_{2}-\left(2 k_{3}+\frac{1}{2} k_{1}^{2}\right) g_{1} \text {, } \\
& \Upsilon_{13}=-\frac{3}{4} k_{1} g_{1}, \\
& \Upsilon_{22}=k_{4} k_{2}+k_{2}^{3}-\left(k_{2}^{2}+2 k_{4}\right) g_{1} \text {, } \\
& \Upsilon_{23}=-k_{2}^{2}-\frac{1}{2} k_{2} g_{1} \text {, } \\
& \Upsilon_{31}=\Upsilon_{13} \text {, } \\
& \Upsilon_{32}=\Upsilon_{23} \text {, } \\
& \Upsilon_{33}=k_{2}
\end{aligned}
$$

Clearly, by using condition (29), we can easily get that $\Theta>0$ and $\Upsilon>0$. Then, it follows from Rayleigh's inequality that

$$
\dot{V} \leq-\frac{1}{\|\xi\|^{1 / 2}} \zeta^{T} \Theta \zeta \leq-\frac{1}{\|\xi\|^{1 / 2}} \lambda_{\min }(\Theta)\|\zeta\|^{2}
$$

By defining $\bar{\zeta}=\operatorname{col}\left(\boldsymbol{\xi}\|\boldsymbol{\xi}\|^{-1 / 2}, \boldsymbol{\xi}, \boldsymbol{z}\right)$, we can rewrite (39) as

$$
\dot{V} \leq-\frac{1}{\|\xi\|^{1 / 2}} \lambda_{\min }(\Theta)\|\bar{\zeta}\|^{2}
$$

Furthermore,

$$
\dot{V} \leq-\frac{1}{\|\xi\|^{1 / 2}} \frac{\lambda_{\min }(\Theta)}{\lambda_{\max }(\Upsilon)} V
$$

Since $V^{1 / 2}>\sqrt{\lambda_{\min }(\Upsilon)}\|\xi\|^{1 / 2}$, it can be proven that

$$
\dot{V} \leq-\frac{\lambda_{\min }(\Theta) \sqrt{\lambda_{\min }(\Upsilon)}}{\lambda_{\max }(\Upsilon)} V^{1 / 2}
$$

According to Lemma 3, it can be observed that the equilibrium point $(\xi, z)=0$ can be reached in finite time. Then by substituting $\boldsymbol{\xi}=\boldsymbol{z}=0$ into (31), we know $\dot{\xi}=0$. As a result, $\xi=\dot{\xi}=0$ is achieved in finite time. Define the estimation error of the mismatched uncertainty as $\widetilde{\boldsymbol{d}}_{\text {mis }}=\widehat{\boldsymbol{d}}_{\text {mis }}-\boldsymbol{d}_{\text {mis }}$. Then from (26), it can be proven that $\dot{\boldsymbol{\xi}}=\widetilde{\boldsymbol{d}}_{\text {mis }}$. Therefore, it can be concluded that $\tilde{\boldsymbol{d}}_{\text {mis }}$ is forced to zero in finite time. The proof is completed.

3.3. Hierarchical Fuzzy Approximation-Based Adaptive FaultTolerant Tracking Control. Considering the following transformed dynamic equation

$$
\begin{aligned}
\dot{s}= & \bar{s}\left(A x_{2}+f_{1}\left(x_{1}, x_{2}\right)+\Delta f_{1}\left(x_{1}, x_{2}\right)+D_{F}-x_{1, r}\right. \\
& -\psi) \\
\dot{x}_{2} & =f_{2}\left(x_{1}, x_{2}\right)+\Delta f_{2}\left(x_{1}, x_{2}\right)+B u+B \Delta \delta+D_{M}
\end{aligned}
$$

we design the virtual control signal as follows:

$$
\begin{aligned}
\boldsymbol{x}_{2 c} & =[\overline{\boldsymbol{s}} \boldsymbol{A}]^{-1} \\
& \cdot\left(-\boldsymbol{K}_{1} \boldsymbol{s}-\overline{\boldsymbol{s}} \boldsymbol{f}_{1}\left(\boldsymbol{x}_{1}, \boldsymbol{x}_{2}\right)-\overline{\boldsymbol{s}} \widehat{\boldsymbol{d}}_{\text {mis }}+\overline{\boldsymbol{s}} \dot{x}_{1, \boldsymbol{r}}+\overline{\boldsymbol{s}} \psi\right)
\end{aligned}
$$

where $K_{1} \in \mathbb{R}^{3 \times 3}$. To avoid the excessive complexity of control design, a first-order filter is established as

$$
\boldsymbol{\tau} \dot{\boldsymbol{x}}_{2 d}+\boldsymbol{x}_{2 d}=\boldsymbol{x}_{2 c}-\boldsymbol{\tau} \boldsymbol{A}^{T} \overline{\boldsymbol{s}}^{T} \boldsymbol{s}, \quad \boldsymbol{x}_{2 d}(0)=\boldsymbol{x}_{2 c}(0)
$$

where $\tau \in \mathbb{R}^{3 \times 3}$ is the parameter matrix of the filter. Denote $\boldsymbol{e}_{2}=\boldsymbol{x}_{2}-\boldsymbol{x}_{2 d}, \boldsymbol{y}=\boldsymbol{x}_{2 d}-\boldsymbol{x}_{2 c}$. Then from (43) (45) we can infer the closed-loop dynamic equation as

$$
\begin{aligned}
& \dot{\boldsymbol{s}}=\overline{\boldsymbol{s}} \boldsymbol{A}\left(\boldsymbol{e}_{2}+\boldsymbol{y}\right)-\boldsymbol{K}_{1} \boldsymbol{s}-\overline{\boldsymbol{s}}_{\boldsymbol{m}} \tilde{\boldsymbol{s}}_{\boldsymbol{s}} \\
& \dot{\boldsymbol{y}}=-\frac{1}{\boldsymbol{\tau}} \boldsymbol{y}-\boldsymbol{A}^{T} \overline{\boldsymbol{s}}^{T} \boldsymbol{s}-\dot{\boldsymbol{x}}_{2 c}
\end{aligned}
$$

Accordingly, along (43), take the derivate of $\boldsymbol{e}_{2}$ as

$$
\begin{aligned}
\dot{\boldsymbol{e}}_{2}= & \boldsymbol{f}_{2}\left(\boldsymbol{x}_{1}, \boldsymbol{x}_{2}\right)+\Delta \boldsymbol{f}_{2}\left(\boldsymbol{x}_{1}, \boldsymbol{x}_{2}\right)+\boldsymbol{B} \boldsymbol{u}+\boldsymbol{B} \Delta \boldsymbol{\delta}+\boldsymbol{D}_{M} \\
& -\dot{x}_{2 d}
\end{aligned}
$$




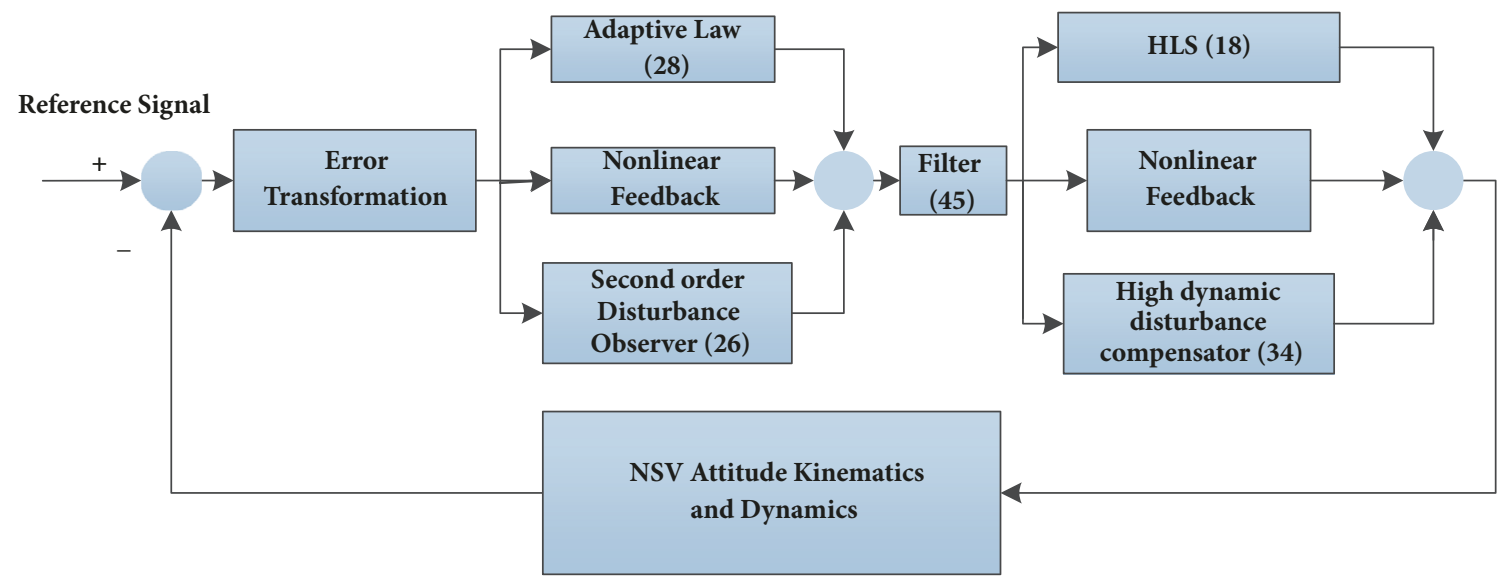

FIGURE 2: The structure of the proposed control method.

In view of the unknown nonlinearities existing in (47), a hierarchical fuzzy logic system $\boldsymbol{W}^{T} \boldsymbol{\Phi}(\boldsymbol{x})$ is constructed for compensation. Ideally, $\Delta \boldsymbol{f}_{2}\left(\boldsymbol{x}_{1}, \boldsymbol{x}_{2}\right)=\boldsymbol{W}^{T} \boldsymbol{\Phi}(\boldsymbol{x})+\boldsymbol{\varepsilon}_{\phi} . \boldsymbol{W}$ is the weight matrix of the HFLS, $N$ is the number of the rules, and $\boldsymbol{\Phi}(\boldsymbol{x}) \in \mathbb{R}^{N}$ is the membership function. The final control law is developed as

$$
\begin{aligned}
\boldsymbol{u} & =\boldsymbol{B}^{-1}\left[-\boldsymbol{K}_{2} \boldsymbol{e}_{2}-\boldsymbol{f}_{2}\left(\boldsymbol{x}_{1}, \boldsymbol{x}_{2}\right)-\widehat{\boldsymbol{W}}^{T} \boldsymbol{\Phi}(\boldsymbol{x})+\dot{\boldsymbol{x}}_{2 d}\right. \\
& \left.-\boldsymbol{A}^{T} \overline{\boldsymbol{s}}^{T} \boldsymbol{s}-\widehat{\bar{D}}_{M} \frac{\boldsymbol{e}_{2}}{\sqrt{\boldsymbol{e}_{2}^{T} \boldsymbol{e}_{2}+\varepsilon_{D}^{2}}}\right]
\end{aligned}
$$

where $\boldsymbol{K}_{2}=\operatorname{diag}\left(k_{21}, k_{22}, k_{23}\right) ; \widehat{\boldsymbol{W}} \in \mathbb{R}^{N \times 3}$ is the estimation of $\boldsymbol{W} . \widehat{\bar{D}}_{M}$ represent the estimations of $\bar{D}_{M}, \bar{D}_{M}=\sup _{t \geq 0} \| \mathbf{D}_{M}+$ $\boldsymbol{B} \Delta \boldsymbol{\delta}+\boldsymbol{\varepsilon}_{\phi} \|$. The adaptive laws are given by

$$
\begin{aligned}
\dot{\overline{\bar{D}}}_{M} & =\eta_{D}\left(\frac{\boldsymbol{e}_{2}^{T} \boldsymbol{e}_{2}}{\sqrt{\boldsymbol{e}_{2}^{T} \boldsymbol{e}_{2}+\varepsilon_{D}^{2}}}-\sigma_{D} \widehat{\bar{D}}_{M}\right) \\
\dot{\widehat{\boldsymbol{W}}} & =\boldsymbol{\Gamma}\left(\boldsymbol{\Phi}(\boldsymbol{x}) \boldsymbol{e}_{2}^{T}-\sigma_{W} \widehat{\boldsymbol{W}}\right)
\end{aligned}
$$

$\sigma_{D}, \eta_{D}, \sigma_{W}>0$ are design constants. $\Gamma>0$ are the gain matrixes of the adaptive laws. The structure of the proposed control method is provided in Figure 2.

Theorem 8. Consider system (43). Suppose the estimation error of the mismatched uncertainty is bounded. Then, by using control laws (44) and (48) and adaptive laws (49), it can be ensured that all signals of the overall closed-loop system are globally uniformly bounded. As a result, the predefined control performance bounds can be guaranteed.

Proof. Consider the following Lyapunov function candidate:

$$
V=V_{1}+V_{2}
$$

$$
\begin{aligned}
& V_{1}=\frac{1}{2} \boldsymbol{s}^{T} \boldsymbol{s}+\frac{1}{2} \boldsymbol{y}^{T} \boldsymbol{y} \\
& V_{2}=\frac{1}{2} \boldsymbol{e}_{2}^{T} \boldsymbol{e}_{2}+\frac{1}{2 \eta_{D}} \widetilde{\bar{D}}_{M} \widetilde{\bar{D}}_{M}+\frac{1}{2} \operatorname{tr}\left(\widetilde{\boldsymbol{W}}^{T} \boldsymbol{\Gamma}^{-\mathbf{1}} \widetilde{\boldsymbol{W}}\right)
\end{aligned}
$$

Then, along (46), the derivative of $V_{1}$ can be taken as

$$
\begin{aligned}
\dot{V}_{1}= & s^{T} \bar{s} A\left(e_{2}+y\right)-s^{T} K_{1} s-s^{T} \bar{s} \tilde{d}_{m i s}-\frac{y^{T} y}{\tau} \\
& -y^{T} A^{T} \bar{s}^{T} s-y^{T} \dot{x}_{2 c}
\end{aligned}
$$

Resorting to Young's inequality, it can be proven that

$$
\begin{aligned}
-\boldsymbol{y}^{T} \dot{\boldsymbol{x}}_{2 c} & \leq \frac{\boldsymbol{y}^{T} \boldsymbol{y}}{2 \boldsymbol{\tau}}+\boldsymbol{\tau} \varepsilon_{c}^{2} \\
-\boldsymbol{s}^{T} \overline{\boldsymbol{s}} \tilde{\boldsymbol{d}}_{m i s} & \leq \frac{1}{4} \boldsymbol{s}^{T} \boldsymbol{s}+\overline{\boldsymbol{\varepsilon}}_{m i s}^{2}
\end{aligned}
$$

where $\bar{\varepsilon}_{\text {mis }}=\sup _{t \geq 0}\left\|\overline{\boldsymbol{s}} \widetilde{\boldsymbol{d}}_{\text {mis }}\right\|, \varepsilon_{c}=\sup _{t \geq 0}\left\|\dot{\boldsymbol{x}}_{2 c}\right\|$. Therefore, we can rewrite (51) as

$$
\dot{V}_{1}=\boldsymbol{s}^{T} \overline{\boldsymbol{s}} A \boldsymbol{e}_{2}-\boldsymbol{s}^{T}\left(\boldsymbol{K}_{1}-\frac{1}{4} I\right) \boldsymbol{s}-\frac{\boldsymbol{y}^{T} \boldsymbol{y}}{2 \tau}+\varepsilon_{1}
$$

where

$$
\varepsilon_{1}=\bar{\varepsilon}_{m i s}^{2}+\|\boldsymbol{\tau}\| \varepsilon_{c}^{2}
$$

From (47) and (48), it can be obtained that

$$
\begin{aligned}
\dot{\boldsymbol{e}}_{2}= & -\boldsymbol{K}_{2} \boldsymbol{e}_{2}-\widehat{\bar{D}}_{M} \frac{\boldsymbol{e}_{2}}{\sqrt{\boldsymbol{e}_{2}^{T} \boldsymbol{e}_{2}+\varepsilon_{D}^{2}}}-\widetilde{\boldsymbol{W}}^{T} \boldsymbol{\Phi}(\boldsymbol{x})-\boldsymbol{A}^{T} \overline{\boldsymbol{s}}^{T} \boldsymbol{s} \\
& +\boldsymbol{B} \Delta \boldsymbol{\delta}+\boldsymbol{D}_{M}+\boldsymbol{\varepsilon}_{\phi}
\end{aligned}
$$


where $\widetilde{\boldsymbol{G}}=\widehat{\boldsymbol{G}}-\mathbf{G}, \widetilde{D}_{\omega}=\widehat{\bar{D}}_{\omega}-\bar{D}_{\omega}$. Take the derivative of $V_{2}$ along (55) as

$$
\begin{aligned}
\dot{V}_{2}= & -\boldsymbol{e}_{2}^{T} \boldsymbol{K}_{2} \boldsymbol{e}_{2}-\boldsymbol{e}_{2}^{T} \boldsymbol{A}^{T} \overline{\boldsymbol{s}}^{T} \boldsymbol{s}-\boldsymbol{e}_{2}^{T} \widehat{\overline{\bar{D}}}_{\omega} \frac{\boldsymbol{e}_{2}}{\sqrt{\boldsymbol{e}_{2}^{T} \boldsymbol{e}_{2}+\varepsilon_{D}^{2}}} \\
& -\boldsymbol{e}_{2}^{T} \widetilde{\boldsymbol{W}}^{T} \boldsymbol{\Phi}(\boldsymbol{x})+\boldsymbol{e}_{2}^{T}\left(\boldsymbol{B} \Delta \boldsymbol{\delta}+\boldsymbol{D}_{M}+\boldsymbol{\varepsilon}_{\phi}\right) \\
& +\frac{1}{\eta_{D}} \widetilde{\bar{D}}_{M} \dot{\overline{\bar{D}}}_{M}+\operatorname{tr}\left(\widetilde{\boldsymbol{W}}^{T} \boldsymbol{\Gamma}^{-1} \dot{\boldsymbol{W}}\right)
\end{aligned}
$$

By using Lemma 4, we can get that

$$
\begin{aligned}
\boldsymbol{e}_{2}^{T}\left(\boldsymbol{B} \Delta \boldsymbol{\delta}+\boldsymbol{D}_{M}+\boldsymbol{\varepsilon}_{\phi}\right) & \leq\left\|\boldsymbol{e}_{2}\right\| \bar{D}_{M} \\
& \leq \frac{\bar{D}_{M} \boldsymbol{e}_{2}^{T} \boldsymbol{e}_{2}}{\sqrt{\boldsymbol{e}_{2}^{T} \boldsymbol{e}_{2}+\varepsilon_{D}^{2}}}+\bar{D}_{M} \varepsilon_{D}
\end{aligned}
$$

Further

$$
\begin{gathered}
-\boldsymbol{e}_{2}^{T} \widehat{\overline{\boldsymbol{D}}}_{M} \frac{\boldsymbol{e}_{2}}{\sqrt{\boldsymbol{e}_{2}^{T} \boldsymbol{e}_{2}+\varepsilon_{D}^{2}}}+\boldsymbol{e}_{2}^{T}\left(\boldsymbol{B} \Delta \boldsymbol{\delta}+\boldsymbol{D}_{M}+\boldsymbol{\varepsilon}_{\phi}\right) \\
\leq-\frac{\widetilde{\bar{D}}_{M} \boldsymbol{e}_{2}^{T} \boldsymbol{e}_{2}}{\sqrt{\boldsymbol{e}_{2}^{T} \boldsymbol{e}_{2}+\varepsilon_{D}^{2}}}+\bar{D}_{M} \varepsilon_{D}
\end{gathered}
$$

Combining (56) (58) yields

$$
\begin{aligned}
\dot{V}_{2} \leq & -\boldsymbol{e}_{2}^{T} \boldsymbol{K}_{2} \boldsymbol{e}_{2}-\boldsymbol{e}_{2}^{T} \boldsymbol{A}^{T} \overline{\boldsymbol{s}}^{T} \boldsymbol{s}-\frac{\widetilde{\bar{D}}_{\omega} \boldsymbol{e}_{2}^{T} \boldsymbol{e}_{2}}{\sqrt{\boldsymbol{e}_{2}^{T} \boldsymbol{e}_{2}+\varepsilon_{D}^{2}}} \\
& -\boldsymbol{e}_{2}^{T} \widetilde{\boldsymbol{W}}^{T} \boldsymbol{\Phi}(\boldsymbol{x})+\frac{1}{\eta_{D}} \widetilde{\bar{D}}_{M} \dot{\overline{\bar{D}}}_{M}+\operatorname{tr}\left(\widetilde{\boldsymbol{W}}^{T} \boldsymbol{\Gamma}^{-1} \dot{\widehat{\boldsymbol{W}}}\right) \\
& +\bar{D}_{M} \mathcal{\varepsilon}_{D}
\end{aligned}
$$

By using the vector trace identity $\boldsymbol{a}^{T} \boldsymbol{b}=\operatorname{tr}\left(\boldsymbol{b} \boldsymbol{a}^{T}\right)$ we can obtain that

$$
\begin{aligned}
\dot{V}_{2} \leq & -\boldsymbol{e}_{2}^{T} \boldsymbol{K}_{2} \boldsymbol{e}_{2}-\boldsymbol{e}_{2}^{T} \boldsymbol{A}^{T} \overline{\boldsymbol{s}}^{T} \boldsymbol{s}-\frac{\widetilde{\bar{D}}_{\omega} \boldsymbol{e}_{2}^{T} \boldsymbol{e}_{2}}{\sqrt{\boldsymbol{e}_{2}^{T} \boldsymbol{e}_{2}+\varepsilon_{D}^{2}}} \\
& -\boldsymbol{e}_{2}^{T} \widetilde{\boldsymbol{W}}_{2}^{T} \boldsymbol{\Phi}_{2}(\boldsymbol{x})+\frac{1}{\eta_{D}} \widetilde{\bar{D}}_{\omega} \dot{\overline{\bar{D}}}_{\omega} \\
& +\operatorname{tr}\left(\widetilde{\boldsymbol{W}}^{T}\left(\boldsymbol{\Gamma}^{-1} \widehat{\boldsymbol{W}}-\boldsymbol{\Phi}(\boldsymbol{x}) \boldsymbol{e}_{2}^{T}\right)\right)+\bar{D}_{M} \boldsymbol{\varepsilon}_{D}
\end{aligned}
$$

Subsisting (49) into (60), we come to

$$
\begin{aligned}
\dot{V}_{2} \leq & -\boldsymbol{e}_{2}^{T} \boldsymbol{K}_{2} \boldsymbol{e}_{2}-\boldsymbol{e}_{2}^{T} \boldsymbol{A}^{T} \overline{\boldsymbol{s}}^{T} \boldsymbol{s}-\sigma_{D} \widetilde{\bar{D}}_{M} \widehat{\bar{D}}_{M} \\
& -\sigma_{W} \operatorname{tr}\left(\widetilde{\boldsymbol{W}}^{T} \widehat{\boldsymbol{W}}\right)+\bar{D}_{M} \boldsymbol{\varepsilon}_{D}
\end{aligned}
$$

Then it follows from (53) and (61) that

$$
\begin{aligned}
\dot{V} \leq & -\boldsymbol{e}_{1}^{T}\left(\boldsymbol{K}_{1}-\frac{1}{4} \boldsymbol{I}\right) \boldsymbol{e}_{1}-\boldsymbol{e}_{2}^{T} \boldsymbol{K}_{2} \boldsymbol{e}_{2}-\frac{\boldsymbol{y}^{T} \boldsymbol{y}}{2 \boldsymbol{\tau}} \\
& -\sigma_{W} \operatorname{tr}\left(\widetilde{\boldsymbol{W}}^{T} \widehat{\boldsymbol{W}}\right)-\sigma_{D} \widetilde{\bar{D}}_{M} \widehat{\bar{D}}_{M}+\varepsilon_{1}+\bar{D}_{M} \varepsilon_{D}
\end{aligned}
$$

Clearly,

$$
\begin{aligned}
-\widetilde{\boldsymbol{W}}^{T} \widehat{\boldsymbol{W}} & \leq-\frac{1}{2} \widetilde{\boldsymbol{W}}^{T} \widetilde{\boldsymbol{W}}+\frac{1}{2} \boldsymbol{W}^{T} \boldsymbol{W},-\widetilde{\bar{D}}_{\omega} \widehat{\bar{D}}_{\omega} \\
& \leq-\frac{1}{2} \widetilde{\bar{D}}_{\omega}^{2}+\frac{1}{2} \bar{D}_{\omega}^{2}
\end{aligned}
$$

Therefore, it can be checked that

$$
\begin{aligned}
\dot{V} \leq & -\boldsymbol{e}_{1}^{T}\left(\boldsymbol{K}_{1}-\frac{1}{4} \boldsymbol{I}\right) \boldsymbol{e}_{1}-\boldsymbol{e}_{2}^{T} \boldsymbol{K}_{2} \boldsymbol{e}_{2}-\frac{\boldsymbol{y}^{T} \boldsymbol{y}}{2 \boldsymbol{\tau}} \\
& -\frac{1}{2} \sigma_{W} \operatorname{tr}\left(\widetilde{\boldsymbol{W}}^{T} \widetilde{\boldsymbol{W}}\right)+\frac{1}{2} \sigma_{W} \operatorname{tr}\left(\boldsymbol{W}^{T} \boldsymbol{W}\right) \\
& -\frac{1}{2} \sigma_{D} \widetilde{\bar{D}}_{M}^{2}+\frac{1}{2} \sigma_{D} \bar{D}_{M}^{2}+\varepsilon_{1}+\bar{D}_{M} \varepsilon_{D}
\end{aligned}
$$

Furthermore, the following inequality holds:

$$
\dot{V} \leq-\lambda V+\varepsilon
$$

where

$$
\begin{aligned}
& \lambda=\min \left\{2\left(\boldsymbol{K}_{1}-\frac{1}{4} \boldsymbol{I}\right), 2 \boldsymbol{K}_{2}, \frac{1}{\boldsymbol{\tau}}, \eta_{D} \sigma_{D}, \sigma_{W}\right\} \\
& \varepsilon=\frac{1}{2} \sigma_{W} \operatorname{tr}\left(\boldsymbol{W}^{T} \boldsymbol{W}\right)+\frac{1}{2} \sigma_{D} \bar{D}_{\omega}^{2}+\varepsilon_{1}+\bar{D}_{\omega} \varepsilon_{D}
\end{aligned}
$$

Solving (65) implies that $0 \leq V(t) \leq \max \{\varepsilon / 2 \lambda, V(0)\}$. Therefore, we can conclude that all the signals in the closedloop system are globally ultimately bounded. Moreover, since $s_{i}, i \in\left\{\alpha, \beta, \gamma_{V}\right\}$ are bounded, the predefined control performance bounds can be guaranteed. The proof is complete.

Remark 9. Note that the matched uncertainties and the actuator derivations are quickly time-varying. To handle the high dynamic uncertainties, we design the adaptive laws (49) and introduce a novel nonlinear function given in (14). As a result, the robustness of the proposed method with respect to the time-varying uncertainties can be improved. By estimating the upper bounds of the time-varying matched disturbances, this problem can be addressed. Moreover, as is well known, SMDO possess strong robustness and accuracy to estimate a lumped disturbance including unknown external disturbances and parametric uncertainties. As a further development, the second-order sliding mode disturbance observer can produce continuous estimation signals and possess strong robustness. In the dynamic model of the NSV, the mismatched disturbance cannot be neglected. Therefore, to deal with the mismatched uncertainties $\boldsymbol{d}_{\text {mis }}=\Delta \boldsymbol{f}_{1}\left(\boldsymbol{x}_{1}, \boldsymbol{x}_{2}\right)+$ $\boldsymbol{D}_{\boldsymbol{F}}$ existing in (24), a second-order sliding mode disturbance observer is introduced for estimation and compensation. At last, it should be noted that the modeling errors are also included in the high dynamic matched uncertainties and the mismatched uncertainties. Based on the previous analysis, it can be concluded that the proposed method has strong robustness with respect to the modeling errors.

\section{Simulation Study}

In this section, the proposed adaptive fuzzy tracking controller is used for the attitude system of the NSV and 


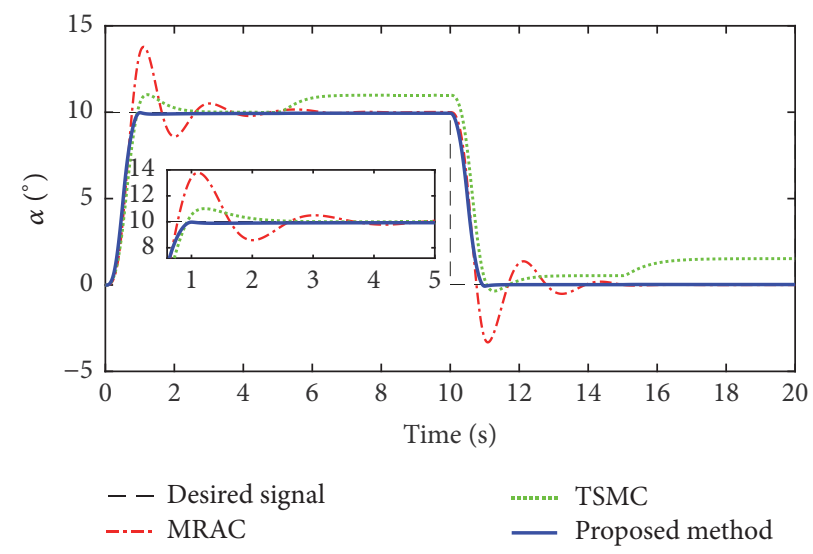

FIGURE 3: The trajectories of angle of attack under the three methods of Case 1.

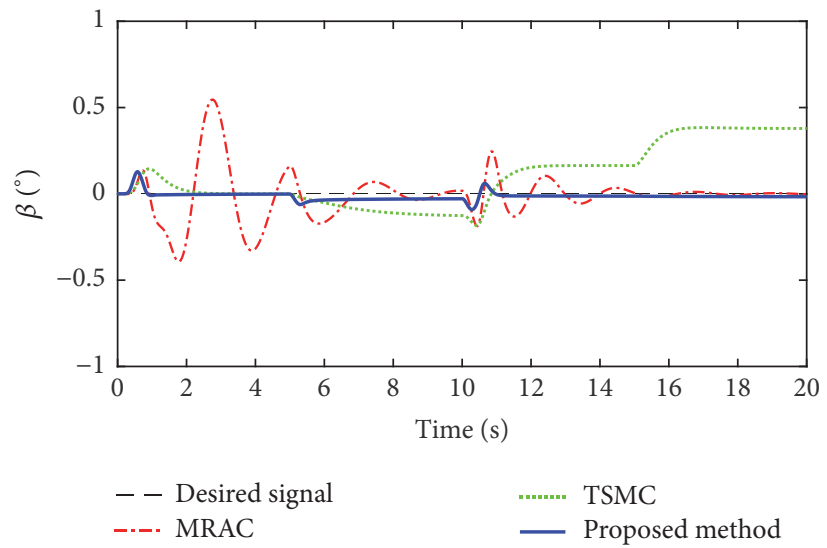

FIgURE 4: The trajectories of sideslip angle under the three methods of Case 1.

simulation results are given to illustrate the effectiveness. The conventional model reference adaptive control method (MRAC) [41] and the terminal sliding mode control method (TSMC) [42] are employed in the simulation to verify the advantages of the proposed method. In the simulation, we consider two cases of the multiple uncertainties and actuator faults.

Firstly, we consider different levels of multiple uncertainties before and after the instant $t=5 \mathrm{~s}$ in the simulation. The unknown winds, which can induce aerodynamic disturbances, are taken into consideration. Let $U$ and $W$ represent the speed of the horizontal wind and the vertical wind, respectively. When $\mathrm{t} \leq 5 s$, the unknown winds are set as $U=30 \mathrm{~m} / \mathrm{s}, W=60 \mathrm{~m} / \mathrm{s}$ and when $t \geq 5 \mathrm{~s}$ they turn to $U=40 \mathrm{~m} / \mathrm{s}, W=70 \mathrm{~m} / \mathrm{s}$. The perturbations of aerodynamic parameters are set as $-20 \%$ when $t \leq 5 \mathrm{~s}$ and $-30 \%$ when $5 s<t \leq 20 s$. The actuator deviations are set as $\left[\begin{array}{lll}0.3 & 0.6 & 0.4\end{array}\right]^{T}$ when $t \leq 5 s$ and $\left[\begin{array}{lll}1.1 & 0.9 & 0.95\end{array}\right]^{T}$ when $5 s<t \leq 20 s$. The parameters of the SOSMDO are set as $k_{1}=7, k_{2}=7, k_{3}=10, k_{4}=10$. Meanwhile, we choose $\boldsymbol{K}_{1}=$ $\operatorname{diag}(3,3,3), \boldsymbol{K}_{2}=\operatorname{diag}(8,8,8)$ and $\sigma_{D}=0.05, \sigma_{W}=0.05$. The filter parameters are set as $\tau=0.08$. Figures $3-8$ provide the simulation results.

The tracking performance of the attitude angles is given in Figures 3-5. It is easy to find that the proposed method
TABLE 1: The parameters of the uncertainties and actuator deviations of Case 2.

\begin{tabular}{lcc}
\hline Variable & $t \leq 5 s$ & $5 s<t \leq 20 s$ \\
\hline$U(m / s)$ & $20+2 \cos (0.5 t)$ & $50+5 \cos (0.5 t)$ \\
$W(m / s)$ & $50+3 \sin (0.2 t)$ & $80+7 \sin (0.2 t)$ \\
$\Delta \delta_{i}$ & $0.05 \cos (0.5 t)$ & $0.25 \cos (0.5 t)$ \\
\hline
\end{tabular}

can achieve satisfactory control performance in the presence of actuator faults and multiple uncertainties. The proposed method can steer the tracking errors to zero, while the MRAC and TSMC methods cannot. The control signals and adaptive parameters are given in Figures 6 and 7, which illustrate the boundness of the signals of the closed-loop system. The estimation results for the mismatched uncertainties via SOSMDO are shown in Figure 8. The exact estimation for the mismatched disturbances improves the control accuracy.

Then we consider the sinusoidal uncertainties and actuator faults. When $t \leq 5 s$ and $5 s<t \leq 20 s$, it is assumed that the aerodynamic parameters possess $0.1 \cos (t)$ and $0.2 \cos (t)$ uncertainties, respectively. The parameters of the other uncertainties and actuator deviations are provided in Table 1. We select the control gains, observation gains, and the adaptive gains as same as Case 1. The simulation results are given in Figures 9-12. It is obvious that the satisfactory attitude 


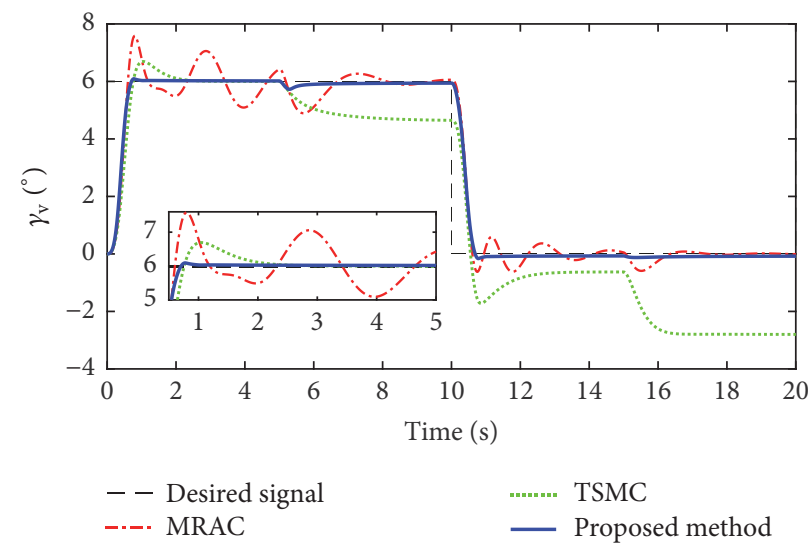

Figure 5: The trajectories of bank angle under the three methods of Case 1.
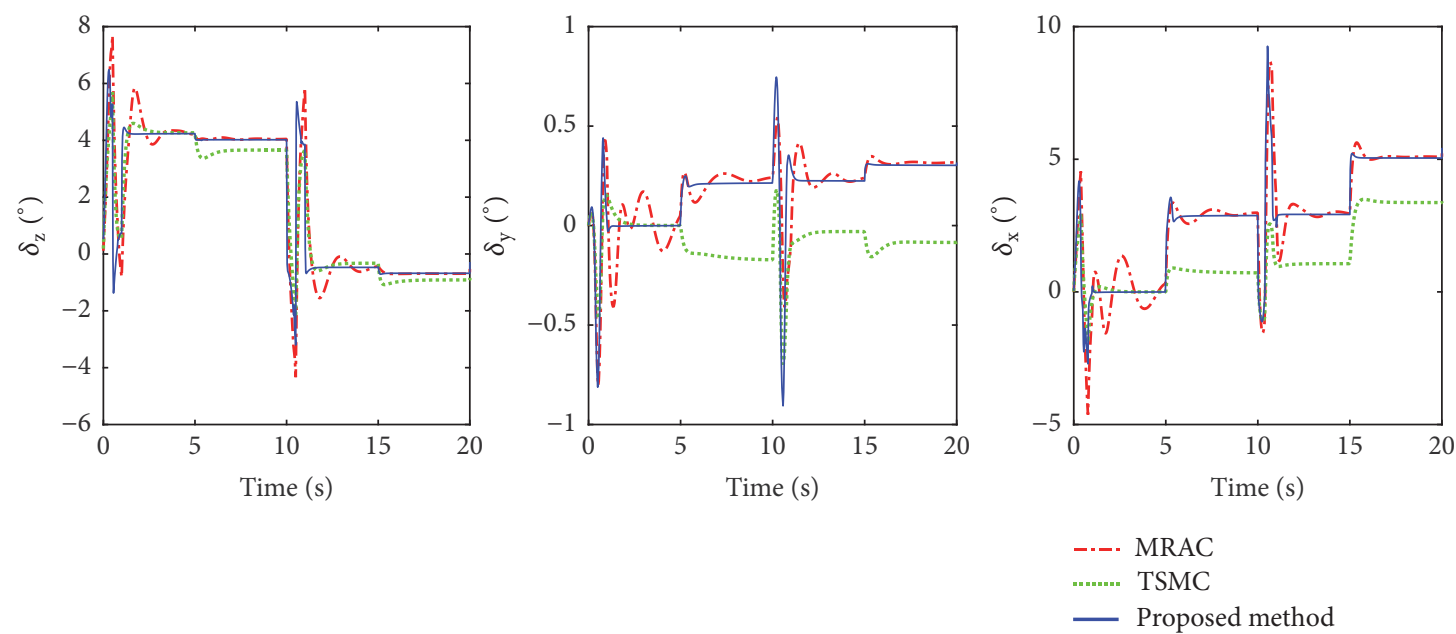

FiguRE 6: The trajectories of the control signals under the three methods of Case 1.
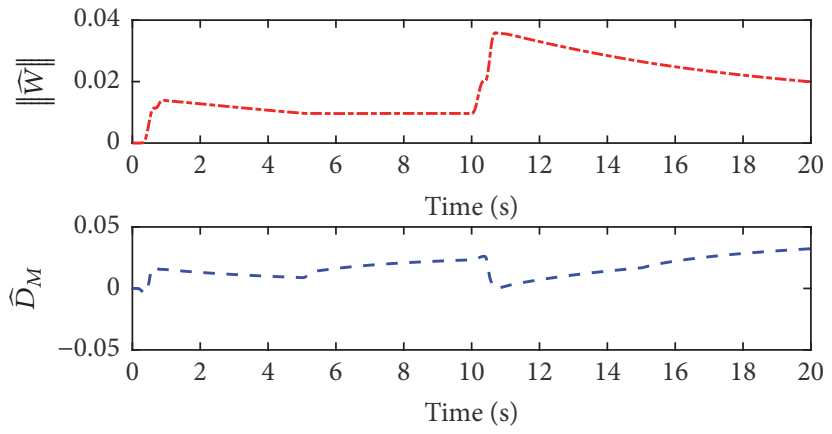

FIGURE 7: The trajectories of the adaptive parameters of Case 1.

tracking performance can also be achieved when the NSV suffers from sinusoidal external disturbances. The strong robustness of the proposed method with respect to the different kinds of multiple uncertainties is demonstrated therefore.

\section{Conclusions}

In this paper, an adaptive tracking controller based on hierarchical fuzzy approximator and adaptive bound estimation approach has been developed to achieve high-performance attitude control for the NSV. By introducing a second-order sliding mode disturbance observer, the time-varying mismatched uncertainties can be estimated and compensated. A new nonlinear function is employed to handle the high dynamic matched uncertainties. By using the performance functions and transforming the original system into one incorporating the desired performance criteria, the predefined tracking performance can be guaranteed. Finally, 


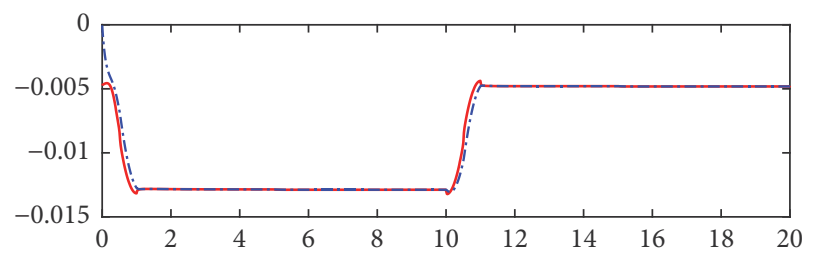

$-\mathrm{d}_{\text {mis }, \alpha}$

-.-. The estimation of $\mathrm{d}_{\text {mis }, \alpha}$

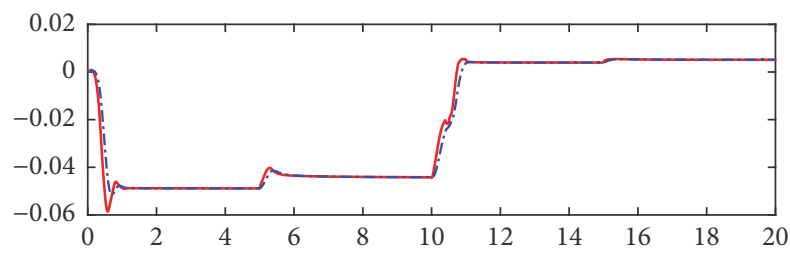

$-\mathrm{d}_{\text {mis, } \beta}$

-.-. The estimation of $\mathrm{d}_{\text {mis, } \beta}$

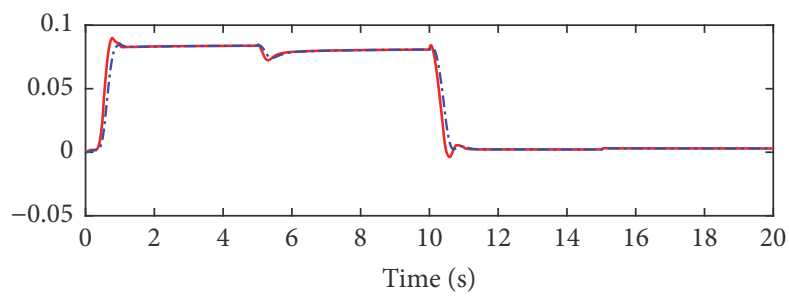

$-\mathrm{d}_{\text {mis, } \gamma_{\mathrm{v}}}$

-.-. The estimation of $\mathrm{d}_{\text {mis }, \gamma_{\mathrm{v}}}$

FIGURE 8: The estimation results of the mismatched uncertainties using SOSMDO.

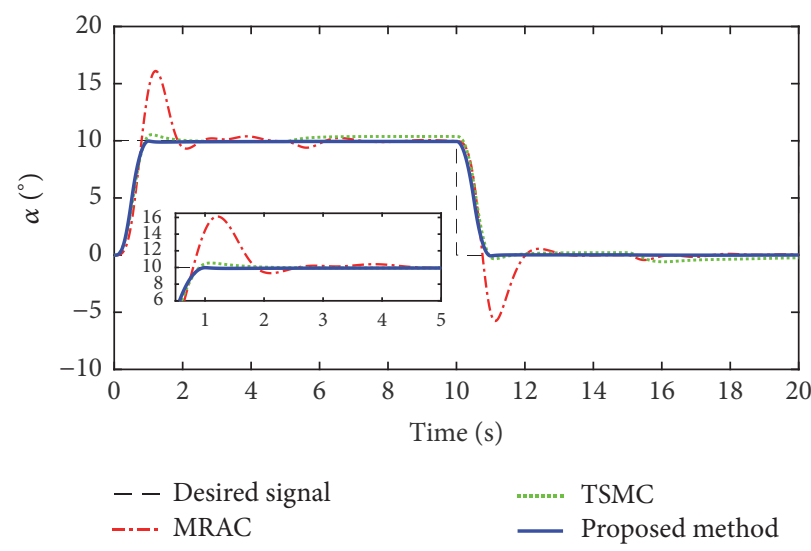

Figure 9: The trajectories of angle of attack under the three methods of Case 2.

simulation results illustrate that the proposed control scheme can achieve satisfactory performance under the disturbance environment.

\section{Data Availability}

The dynamic model of NSV used to support the findings of this study is available from the corresponding author upon request. It can also be found in the reference literature [39].

\section{Conflicts of Interest}

The authors declare that they have no conflicts of interest.

\section{Acknowledgments}

This work was supported by the National Natural Science Foundation of China (no. 61503302 and no. 61473226). 


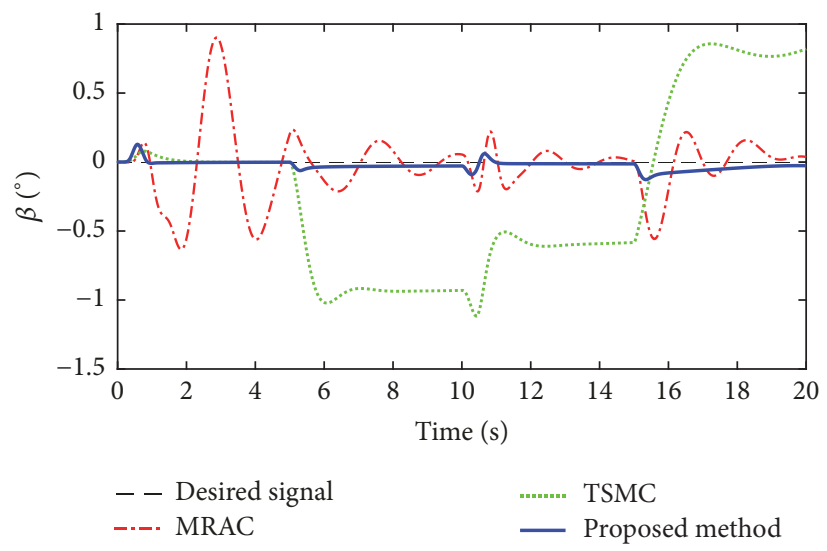

FIgURE 10: The trajectories of sideslip angle under the three methods of Case 2.

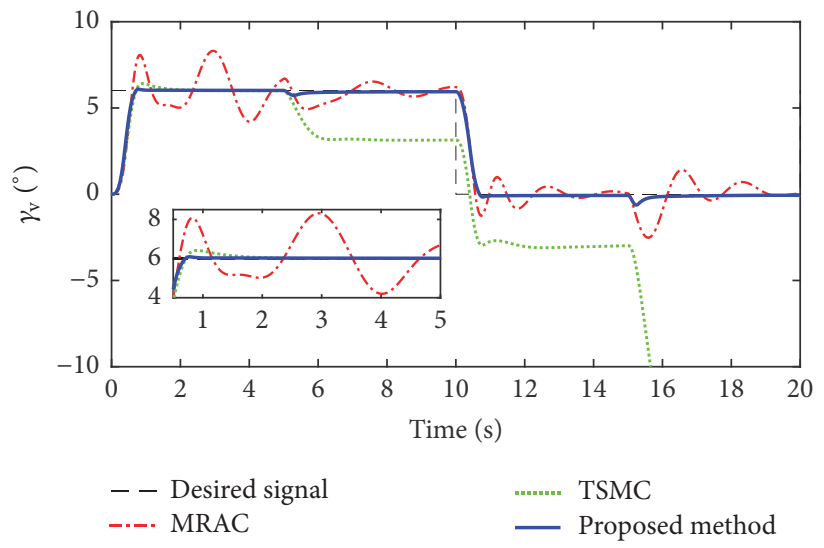

FIGURE 11: The trajectories of bank angle under the three methods of Case 2.
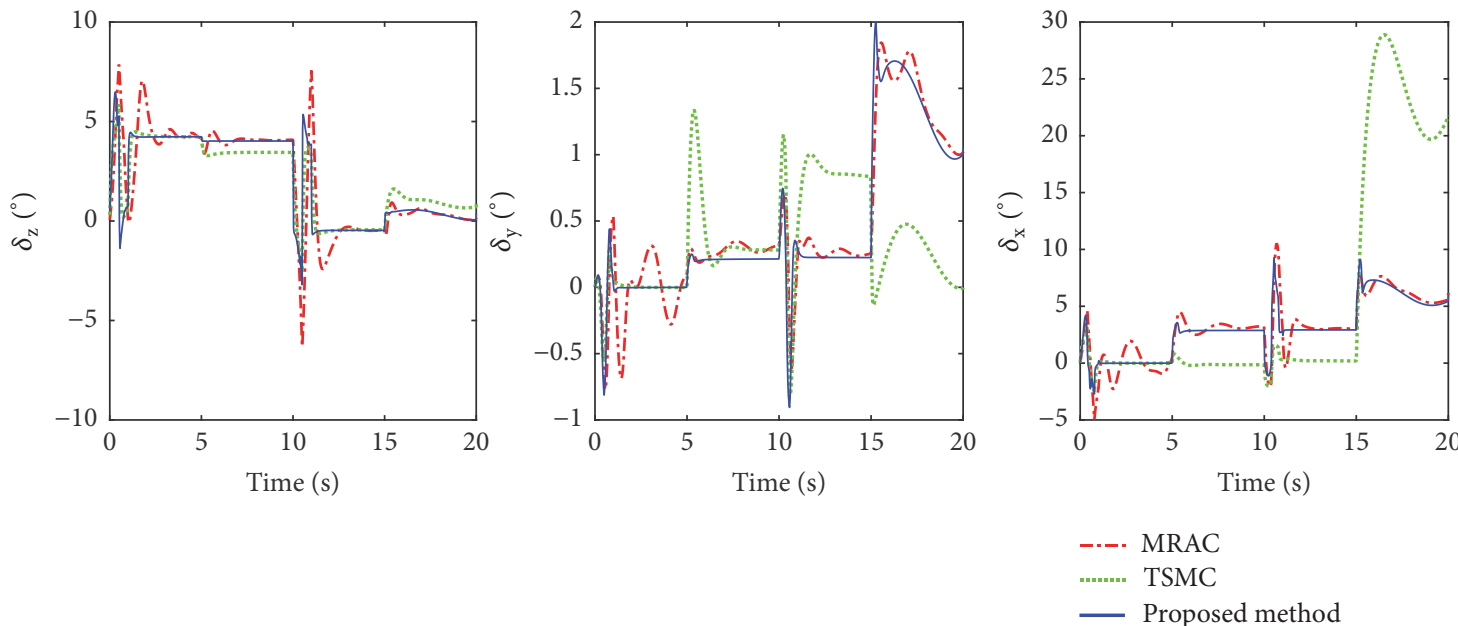

FIGURE 12: The trajectories of the control signals under the three methods of Case 2.

\section{References}

[1] W. Chen, J. Yang, L. Guo, and S. Li, "Disturbance observer-based control and related methods: an overview," IEEE Transactions on Industrial Electronics, vol. 63, no. 2, pp. 1083-1095, 2015.

[2] C. D. Johnson, "Accommodation of External Disturbances in Linear Regulator and Servomechanism Problems," IEEE
Transactions on Automatic Control, vol. 16, no. 6, pp. 635-644, 1971.

[3] C. D. Johnson, "Optimal Control of the Linear Regulator with Constant Disturbances," IEEE Transactions on Automatic Control, vol. 13, no. 4, pp. 416-421, 1968.

[4] S. J. Kwon and K. C. Wan, "Discrete-time design and analysis of perturbation observer," IEEE Transactions on 
Control Systems Technology, vol. 11, no. 3, pp. 399-407, 2003.

[5] Q.-C. Zhong, A. Kuperman, and R. K. Stobart, "Design of UDEbased controllers from their two-degree-of-freedom nature," International Journal of Robust and Nonlinear Control, vol. 21, no. 17, pp. 1994-2008, 2011.

[6] W.-H. Chen, D. J. Ballance, P. J. Gawthrop, and J. O’Reilly, "A nonlinear disturbance observer for robotic manipulators," IEEE Transactions on Industrial Electronics, vol. 47, no. 4, pp. 932-938, 2000.

[7] K. Ohishi, “Torque-speed regulation of DC motor based on load torque estimation," Proc Ipec83, vol. 2, 1983.

[8] H. Muramatsu and S. Katsura, "Adaptive Periodic-Disturbance Observer for Periodic-Disturbance Suppression," IEEE Transactions on Industrial Informatics, pp. 1-1.

[9] D. Lee, "Nonlinear disturbance observer-based robust control for spacecraft formation flying," Aerospace Science and Technology, vol. 76, pp. 82-90, 2018.

[10] Y. Yu, Z. Yang, C. Han, and H. Liu, "Disturbance-observer based control for magnetically suspended wheel with synchronous noise," Control Engineering Practice, vol. 72, pp. 83-89, 2018.

[11] Y. Hu, P. Huang, Z. Meng, D. Wang, and Y. Lu, "Approaching control for tethered space robot based on disturbance observer using super twisting law," Advances in Space Research, vol. 61, no. 9, pp. 2344-2351, 2018.

[12] Z. Wang, J. Yuan, and J. Wei, "Adaptive output feedback disturbance attenuation control for nonlinear systems with non-harmonic multisource disturbances," Optik - International Journal for Light and Electron Optics, vol. 137, pp. 85-95, 2017.

[13] Z. Wang and J. Yuan, "Non-linear disturbance observer-based adaptive composite anti-disturbance control for non-linear systems with dynamic non-harmonic multisource disturbances," Transactions of the Institute of Measurement and Control, p. 014233121772196, 2017

[14] J. Han, "The Extended State Observer of a Class of Uncertain Systems," Control Decis, 1995.

[15] K. D. Young, V. I. Utkin, and U. Ozguner, "A Control Engineers Guide to Sliding Mode Control," IEEE Transactions on Control Systems Technology, vol. 7, no. 3, pp. 328-342, 1999.

[16] J. Zhang, P. Shi, and W. Lin, "Extended sliding mode observer based control for Markovian jump linear systems with disturbances," Automatica, vol. 70, pp. 140-147, 2016.

[17] G. Palli, S. Strano, and M. Terzo, "Sliding-mode observers for state and disturbance estimation in electro-hydraulic systems," Control Engineering Practice, vol. 74, pp. 58-70, 2018.

[18] S. D. Nguyen, S. Choi, and Q. H. Nguyen, "A new fuzzydisturbance observer-enhanced sliding controller for vibration control of a train-car suspension with magneto-rheological dampers," Mechanical Systems and Signal Processing, vol. 105, pp. 447-466, 2018.

[19] H. An, J. Liu, C. Wang, and L. Wu, "Disturbance ObserverBased Antiwindup Control for Air-Breathing Hypersonic Vehicles," IEEE Transactions on Industrial Electronics, vol. 63, no. 5, pp. 3038-3049, 2016.

[20] H. An, C. Wang, and B. Fidan, "Sliding mode disturbance observer-enhanced adaptive control for the air-breathing hypersonic flight vehicle," Acta Astronautica, vol. 139, pp. 111121, 2017.

[21] Y.-S. Lu, C.-M. Cheng, and C.-H. Cheng, "Non-overshooting PI control of variable-speed motor drives with sliding perturbation observers," Mechatronics, vol. 15, no. 9, pp. 1143-1158, 2005.
[22] Y.-S. Lu, "Sliding-mode disturbance observer with switchinggain adaptation and its application to optical disk drives," IEEE Transactions on Industrial Electronics, vol. 56, no. 9, pp. 37433750, 2009.

[23] R. Zhang, C. Sun, J. Zhang, and Y. Zhou, "Second-order terminal sliding mode control for hypersonic vehicle in cruising flight with sliding mode disturbance observer," Control Theory and Technology, vol. 11, no. 2, pp. 299-305, 2013.

[24] B. Wang, Z. Dong, Y. Yu, G. Wang, and D. Xu, "Static-Errorless Deadbeat Predictive Current Control Using Second-Order Sliding-Mode Disturbance Observer for Induction Machine Drives," IEEE Transactions on Power Electronics, vol. 33, no. 3, pp. 2395-2403, 2018.

[25] H. Xu and P. A. Ioannou, "Robust adaptive control for a class of MIMO nonlinear systems with guaranteed error bounds," Institute of Electrical and Electronics Engineers Transactions on Automatic Control, vol. 48, no. 5, pp. 728-742, 2003.

[26] Z. Wang, J. Yuan, Y. Pan, and D. Che, "Adaptive neural control for high order Markovian jump nonlinear systems with unmodeled dynamics and dead zone inputs," Neurocomputing, vol. 247, pp. 62-72, 2017.

[27] C. P. Bechlioulis and G. A. Rovithakis, "Robust adaptive control of feedback linearizable MIMO nonlinear systems with prescribed performance," Institute of Electrical and Electronics Engineers Transactions on Automatic Control, vol. 53, no. 9, pp. 2090-2099, 2008.

[28] W. Wang and C. Wen, "Adaptive actuator failure compensation control of uncertain nonlinear systems with guaranteed transient performance," Automatica, vol. 46, no. 12, pp. 2082-2091, 2010.

[29] C. P. Bechlioulis and G. A. Rovithakis, "Robust partial-state feedback prescribed performance control of cascade systems with unknown nonlinearities," Institute of Electrical and Electronics Engineers Transactions on Automatic Control, vol. 56, no. 9, pp. 2224-2230, 2011.

[30] C. Wei, J. Luo, Z. Yin, X. Wei, and J. Yuan, "Robust estimationfree decentralized prescribed performance control of nonaffine nonlinear large-scale systems," International Journal of Robust and Nonlinear Control, vol. 28, no. 1, pp. 174-196, 2018.

[31] W.-Q. Wang, "Near-space vehicles: Supply a gap between satellites and airplanes for remote sensing," IEEE Aerospace and Electronic Systems Magazine, vol. 26, no. 4, pp. 4-9, 2011.

[32] M. Chen and J. Yu, "Disturbance observer-based adaptive sliding mode control for near-space vehicles," Nonlinear Dynamics, vol. 82, no. 4, pp. 1671-1682, 2015.

[33] M. Chen, B. Jiang, Q. Wu, and C. Jiang, "Robust control of nearspace vehicles with input backlash-like hysteresis," Proceedings of the Institution of Mechanical Engineers, Part I: Journal of Systems and Control Engineering, vol. 227, no. 8, pp. 635-644, 2013.

[34] Y. L. Du, Q. X. Wu, C. S. Jiang, and J. Wen, "Adaptive functional link network control of near-space vehicles with dynamical uncertainties," Journal of Systems Engineering and Electronics, vol. 21, no. 5, pp. 868-876, 2010.

[35] M. Chen and B. Jiang, "Robust attitude control of near space vehicles with time-varying disturbances," International Journal of Control, Automation, and Systems, vol. 11, no. 1, pp. 182-187, 2013.

[36] Y. Wang, "NSV attitude system fuzzy modeling based on modified L-M algorithm," J. Southeast Univ, vol. 40, pp. 103-108, 2010. 
[37] M. Pu, Q. Wu, C. Jiang, and L. Cheng, "fast terminal sliding mode and its application to near space vehicles," Acta Aeronaut. Astronaut. Sin, vol. 32, no. 7, pp. 1283-1291, 2011.

[38] J. Valasek, D. Ito, and D. Ward, "Robust dynamic inversion controller design and analysis for the X-38," in Proceedings of the AIAA Guidance, Navigation, and Control Conference and Exhibit, Montreal,Canada.

[39] S. Keshmiri, M. D. Mirmirani, and R. D. Colgren, "Six-DOF modeling and simulation of a generic hypersonic vehicle for conceptual design studies," in Proceedings of the Collection of Technical Papers - AIAA Modeling and Simulation Technologies Conference, pp. 98-109, usa, August 2004.

[40] L.-X. Wang, "Analysis and design of hierarchical fuzzy systems," IEEE Transactions on Fuzzy Systems, vol. 7, no. 5, pp. 617-624, 1999.

[41] C. Wei, J. Luo, H. Dai, and J. Yuan, "Adaptive modelfree constrained control of postcapture flexible spacecraft: a Euler-Lagrange approach," Journal of Vibration and Control, p. 107754631773696, 2017.

[42] L. Guo and S. Cao, "Anti-disturbance control theory for systems with multiple disturbances: a survey," ISA Transactions ${ }^{\circledR}$, vol. 53, no. 4, pp. 846-849, 2014. 


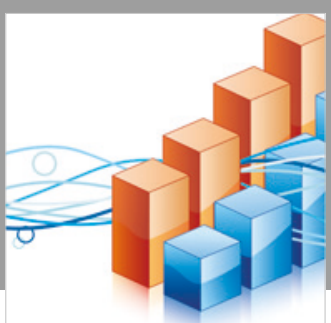

Advances in

Operations Research

\section{-n-m}
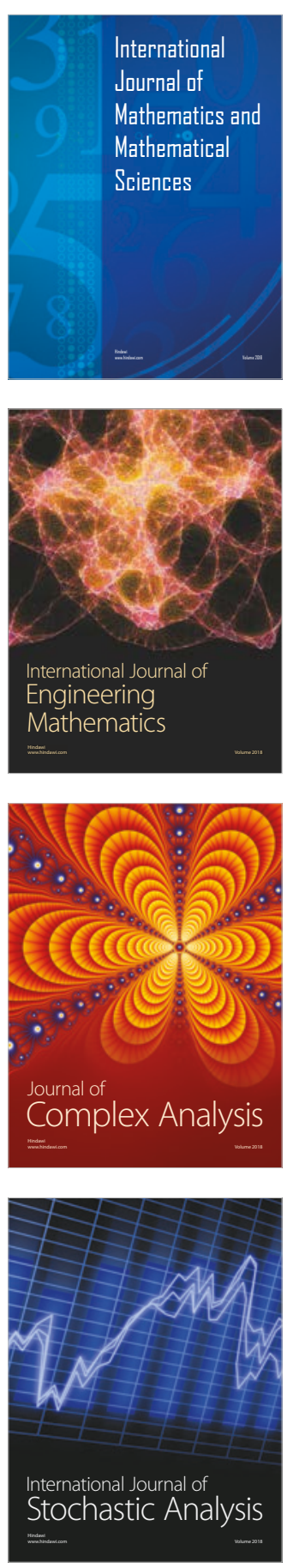
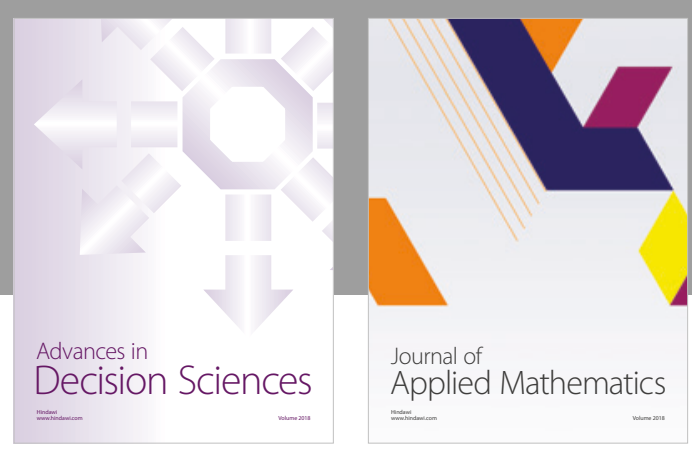

Journal of

Applied Mathematics
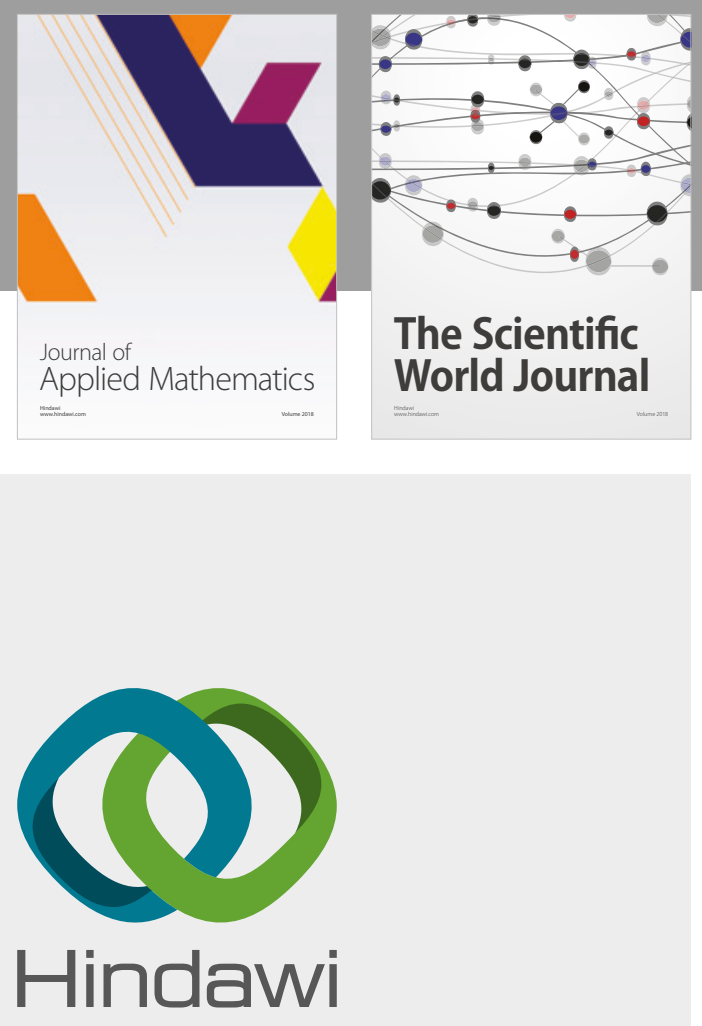

Submit your manuscripts at

www.hindawi.com

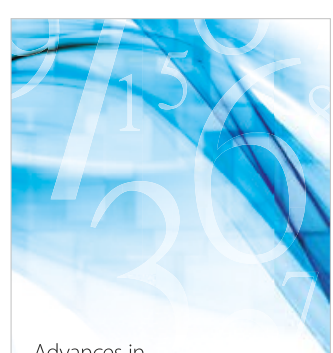

Advances in
Numerical Analysis
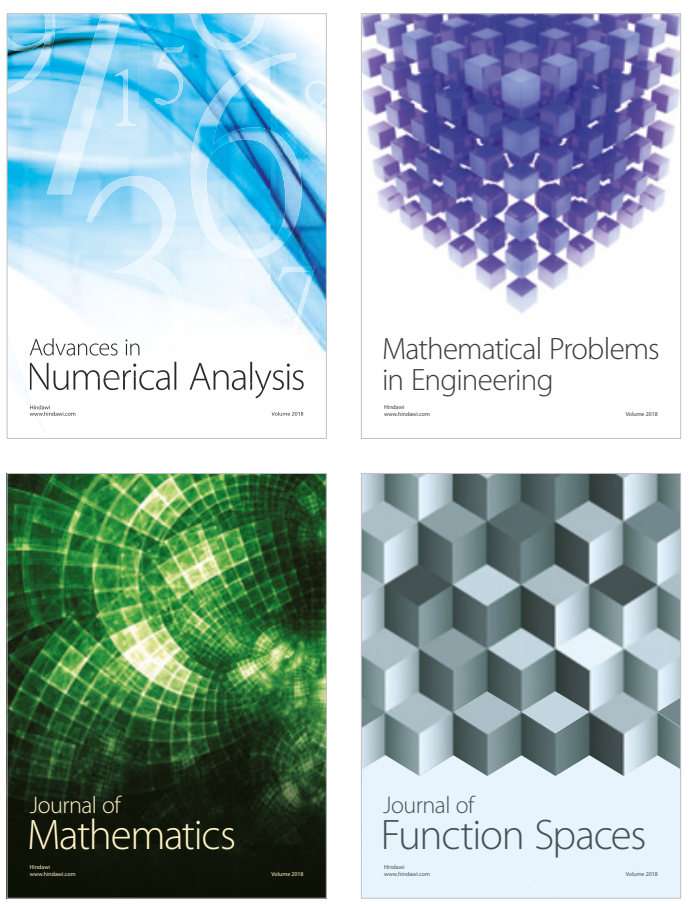

Mathematical Problems in Engineering

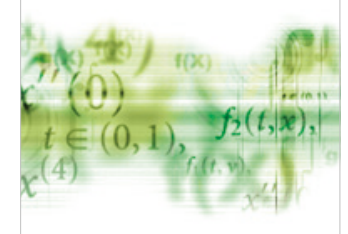

International Journal of

Differential Equations

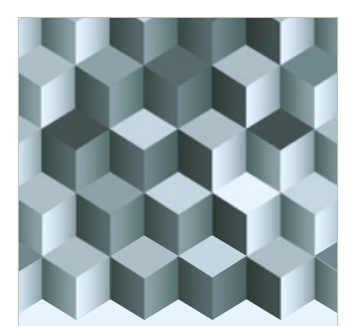

Journal of

Function Spaces

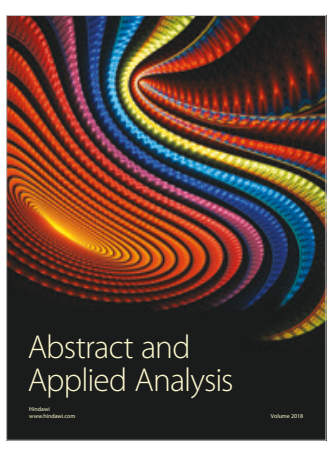

The Scientific

World Journal

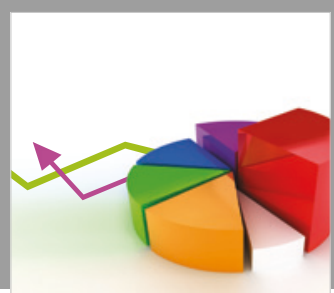

Journal of

Probability and Statistics
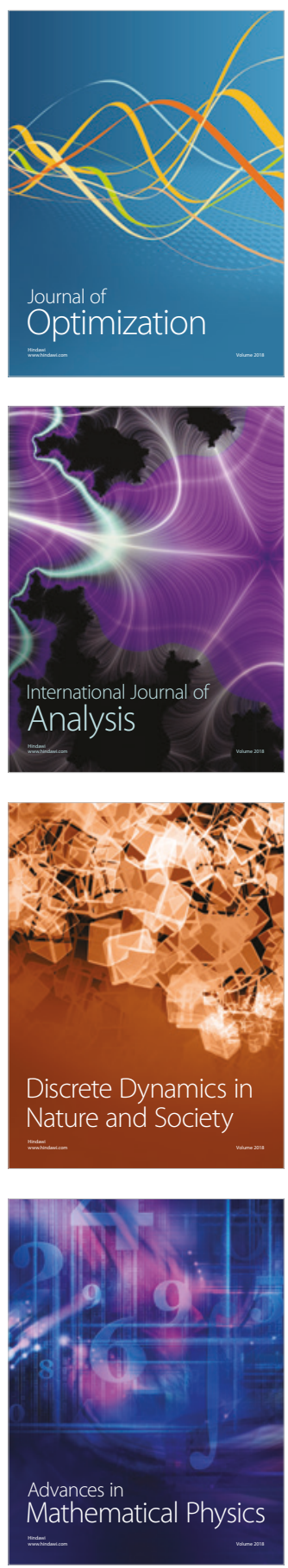